\title{
A GENERALIZED OPERATIONAL CALCULUS DEVELOPED FROM FREDHOLM OPERATOR THEORY(1)
}

\author{
BY
}

\section{JACK SHAPIKO AND MARTIN SCHECHTER (2)}

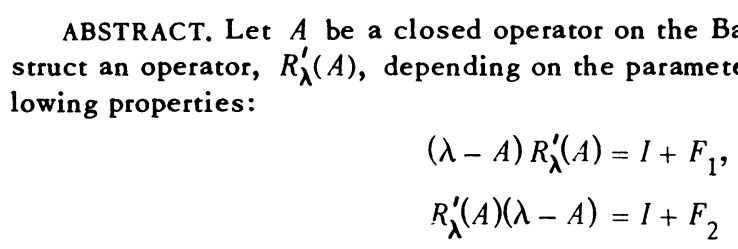

where $F_{1}$ and $F_{2}$ are bounded finite rank operators. $R_{\lambda}^{\prime}(A)$ is defined and analytic in $\lambda$ for all $\lambda \in \Phi_{A}$ except for at most a countable set containing no accumulation point in $\Phi A^{\text {. }}$

Let $\sigma_{\Phi}(A)$ be the complement of $\Phi_{A}$, and let $f \in \mathbb{Q}_{\infty}^{\prime}(A)$, where $\mathfrak{Q}_{\infty}^{\prime}(A)$ denotes the set of complex valued functions which are analytic on $\sigma_{\Phi}(A)$ and at $(\infty)$. We then use the operator, $R_{\lambda}^{\prime}(A)$, to construct an operational calculus for $A . f(A)$ is defined up to addition by a compact operator. We prove for our operational calculus analogues of the theorems for the classical operational calculus. We then extend a theorem of Kato by using the operator, $R_{\lambda}^{\prime}(A)$, to construct an analytic basis for $N(A-\lambda)$.

1. Introduction. In [6], Taylor developed an operational calculus for closed operators. If $A$ is a closed operator and $f(\lambda)$ is analytic on $\sigma(A), f(A)$ is then defined by $f(A)=(1 / 2 \pi i) \int_{+B(D)} f(\lambda) R_{\lambda}(A) d \lambda$ where $D$ is a suitable domain and $R_{\lambda}(A)$ is the resolvent operator. In this paper we develop an operational calculus which extends the set of functions which can be applied to the operator $A$.

Let $A$ be a densely defined closed linear operator from a Banach space $X$ to a Banach space $Y$.

Definition 1. $A$ is called a Fredholm operator if

(1) $a(A)=\operatorname{dim}[N(A)]<\infty$,

(2) $R(A)$, the range of $A$, is closed in $Y$,

(3) $\beta(A)$, the codimension of $R(A)$ in $Y$, is finite.

We denote the set of Fredholm operators from $X$ to $X$ by $\Phi(X)$.

Presented to the Society, March 8, 1972; received by the editors November 12, 1971. AMS (MOS) subject classifications (1970). Primary 47630; Secondary 47A60, 47B05.

Key words and phrases. Fredholm operator, operational calculus, analytic basis.

(1) This paper contains portions of the doctoral dissertation of Jack Shapiro submitted in partial fulfillment of the requirements for the degree of Doctor of Philosophy at the Belfer Graduate School of Science, Yeshiva University.

(2) Supported by National Science Foundation grant GṔP-19757A1. 
Definition 2. $\lambda \in \Phi_{A}$ if and only if $(\lambda-A) \in \Phi(X)$.

Definition 3. $\lambda \in \sigma_{\Phi}(A)$ if and only if $(\lambda-A) \notin \Phi(X)$.

Let $\mathfrak{Q}_{\infty}^{\prime}(A)$ denote the set of all complex valued functions, $f(\lambda)$, which are analytic on $\sigma_{\Phi}(A)$. In this paper we show how to define an operator $f(A)$ for $f \epsilon$ $\mathbb{Q}_{\infty}^{\prime}(A)$.

Let $L(X)$ denote the set of all bounded operators on $X$.

Definition 4. We shall say that the operator $B \in L(X)$ is a quasi-inverse of the closed operator $A$ if $R(B) \subset D(A)$ and there exist compact operators $K_{1}$ and $K_{2}$ such that $A B=I+K_{1}$ and $B A=\left.\left(I+K_{2}\right)\right|_{D(A)}$.

If $A \in \Phi(X)$, then $A$ has a quasi-inverse. See [3, Lemma 2.3]. We denote the set of all compact operators on $X$ by $\mathcal{K}(X)$.

Let $\Phi_{i}(A)$ be a component of $\Phi_{A}$. For each $\lambda \in \Phi_{i}(A)$ there exists an operator $B_{\lambda} \in L(X)$ such that $(\lambda-A) B_{\lambda}=I+K^{\prime}$ and $B_{\lambda}(\lambda-A)=\left.\left(I+K^{\prime \prime}\right)\right|_{D(A)}$, where $K^{\prime}$ and $K^{\prime \prime}$ are compact operators. If $f(\lambda)$ is analytic on $\sigma_{\Phi}(A)$ we would like to define $f(A)$ by $f(A)=(1 / 2 \pi i) \int_{+B(D)} f(\lambda)_{B_{\lambda}} d \lambda$ where $D$ is a suitable domain and $B_{\lambda}$ is a quasi-inverse of $(\lambda-A)$. However, $B_{\lambda}$, if chosen arbitrarily, is not analytic.

In $\$ 2$ we show how $B_{\lambda}$ can be chosen so that $B_{\lambda}$ is analytic in $\lambda$ throughout $\Phi_{i}(A)$ except for at most a countable set of points having no accumulation point in $\Phi_{i}(A)$. We shall refer to our choice of $B_{\lambda}$ as $R_{\lambda}^{\prime}(A)$. We call $R_{\lambda}^{\prime}(A)$ a quasi-resolvent operator. Although $R_{\lambda}^{\prime}(A)$ can be chosen in different ways, any two choices of $R_{\lambda}^{\prime}(A)$ differ by at most a bounded finite rank operator.

For each $f \in \mathbb{Q}_{\infty}^{\prime}(A)$ we define a class of operators $\mathcal{F}(A)$ in the following way: $B \in \mathcal{F}(A)$ if and only if

$$
B=f(\infty) I+\frac{1}{2 \pi i} \int_{+B(D)} f(\lambda) R_{\lambda}^{\prime}(A) d \lambda .
$$

Different choices of $R_{\lambda}^{\prime}(A)$ and $D$ give different members of $\mathscr{F}(A)$.

In Theorem 7 we show that any two members of $\mathcal{F}(A)$ can differ by at most a compact operator. This compact operator turns out to be the limit of finite rank operators. It should be pointed out here that all the compact operators mentioned in the theorems of this paper turn out to be limits of finite rank operators. We then define $f(A)$ to be an arbitrary member of $\mathcal{F}(A)$.

In Theorems 8 and 9 we show that if $\alpha$ and $\beta$ are complex numbers, and $f$, $g \in \mathbb{Q}_{\infty}^{\prime}(A)$, then $\alpha f(A)+\beta g(A)=(\alpha f+\beta g) A+K_{1}$ and $f(A) \cdot g(A)=(f \cdot g) A+K_{2}$, where $K_{1}$ and $K_{2}$ are compact operators. These compact operators can be computed directly.

In Theorem 10 we show that if $f(\lambda) \equiv 1$, then $f(A)=I+K, K \in K(X)$.

If $A$ is bounded we define $\mathbb{Q}^{\prime}(A)$ as the set of all functions which are analytic on $\sigma_{\Phi}(A)$. If $f \in \mathbb{Q}^{\prime}(A)$, we then define the class of operators $\mathcal{F}^{*}(A)$ as follows: 
$B \in \mathfrak{F}^{*}(A)$ if and only if

$$
B=\frac{1}{2 \pi i} \int_{+B(D)} f(\lambda) R_{\lambda}^{\prime}(A) d \lambda
$$

where $D$ is a suitable bounded domain. We then define $f^{*}(A)$ to be an arbitrary member of $\mathfrak{F}^{*}(A)$. We then prove analogues of the above theorems for $f^{*}(A)$. Theorem 11 shows that if $A$ is bounded and $f \in \mathbb{Q}_{\infty}^{\prime}(A)$, then $f^{*}(A)=f(A)$.

We wish to point out here that if $A$ is a bounded operator one can construct an operational calculus in the following way. Let $Q$ be the quotient algebra $L(X) / K(X)$. Let $[A]$ be the image of $A$ under the natural homomorphism mapping $L(X)$ to $Q$. Then it follows from [3, Lemma 2.3], that $\sigma([A])=\sigma_{\Phi}(A)$. Therefore, if $f \in \mathbb{Q}^{\prime}(A)$, then $f$ must be analytic on $\sigma([A])$. $f([A])$ can then be defined by

$$
f([A])=\frac{1}{2 \pi i} \int_{+B(D)} f(\lambda)(\lambda-[A])^{-1} d \lambda .
$$

$f(A)$ may then be chosen to be any pre-image of $f([A])$ under the natural homomorphism. The disadvantage with this approach is that the particular choice of $f(A)$ is completely arbitrary. In our approach, once we choose a region for integration and make a particular choice of $R_{\lambda}^{\prime}(A), f(A)$ is automatically determined. If $A$ is unbounded, then the first method cannot be applied at all because we cannot construct the quotient algebra.

In Theorem 15 we show that if $f \in \mathbb{Q}_{\infty}^{\prime}(A)$ and if $f$ does not vanish on $\sigma_{\Phi}(A)$, then $f(A)$ has a quasi-inverse in $L(X)$.

In $\$ 3$ we apply the operational calculus to polynomials. Let $P(\lambda)$ be a polynomial in $\lambda$. Since $P(\lambda) \notin \mathbb{Q}_{\infty}^{\prime}(A)$ we cannot define the operator $P(A)$ in the manner described above. If

we define $P(A)$ by

$$
P(\lambda)=\sum_{i=0}^{n} a_{i} \lambda^{i}
$$

$$
P(A)=\sum_{i=0}^{n} a_{i} A^{i}
$$

We then prove the following result in Theorem 1 of this section:

Let $f(\lambda) \in \mathbb{Q}_{\infty}^{\prime}(A)$, and suppose either (a) that $f$ has a zero of order $m$ at $\infty$, or (b) that $f$ vanishes in a neighborhood of $\infty$. Let $P$ be a polynomial of degree $n$, where $0 \leq n \leq m$ in case (a) and $0<n$ in case (b). Let $H(\lambda)=P(\lambda) f(\lambda)$. Then $\forall(H) \in \mathbb{Q}_{\infty}^{\prime}(A), \quad \exists K_{1} \in \mathcal{K}(X)$ such that $R\left[f(A)+K_{1}\right] \subset D\left(A^{n}\right)$ and $\exists K_{2} \in \mathcal{K}(X)$ such that $P(A)\left[f(A)+K_{1}\right]=H(A)+K_{2}$.

Theorem 3 then shows how to express $P(A)$ as an integral.

We now give an example of our operational calculus. Let $H$ be a Hilbert space with orthonormal basis $\left\{e_{i}\right\}_{i=0}^{\infty}$. Let $U$ be the unilateral shift defined by $U e_{i}=e_{i+1}$.

$U^{*}$, the adjoint of $U$, then has the following property. 


$$
\begin{aligned}
U^{*} e_{i} & =e_{i-1} \quad \text { for } i>0, \quad \text { and } \quad U^{*} e_{0}=0, \\
\sigma(U) & =\sigma\left(U^{*}\right)=\{\lambda:|\lambda| \leq 1\} ; \quad \sigma_{\Phi}\left(U^{*}\right)=\{\lambda:|\lambda|=1\}, \\
\left(U^{*} U\right) x & =I, \quad\left(U U^{*}\right) x=\left(I-F_{1}\right) x,
\end{aligned}
$$

where $F_{1}$ is the orthogonal projection onto $\left[e_{0}\right]$, the space spanned by $e_{0}$. Let

$$
P(\lambda)=(\lambda-1 / 2)(\lambda+1 / 2), \quad P\left(U^{*}\right)=\left(U^{*}-1 / 2\right)\left(U^{*}+1 / 2\right) .
$$

We shall construct an operator $B$ such that

$$
B P\left(U^{*}\right)=I+K, \quad K \in K(X) .
$$

Let $f(\lambda)=1 /(\lambda-1 / 2)(\lambda+1 / 2) . f(\lambda)$ is analytic on $\sigma_{\Phi}(A)$ but not on $\sigma(A)$. We define $f\left(U^{*}\right)$ by

$$
\begin{aligned}
f\left(U^{*}\right)= & \frac{1}{2 \pi i} \int_{C_{1}} \frac{1}{\left(\lambda-\frac{1}{2}\right)(\lambda+1 / 2)}(-U)[-\lambda U+I]^{-1} d \lambda \\
& +\frac{1}{2 \pi i} \int_{C_{2}} \frac{1}{(\lambda-1 / 2)(\lambda+1 / 2)}\left(\lambda-U^{*}\right)^{-1} d \lambda
\end{aligned}
$$

where $C_{1}$ is the circle $|\lambda|=3 / 4$ oriented clockwise, and $C_{2}$ is the circle $|\lambda|=2$ oriented counterclockwise.

$$
\begin{gathered}
\frac{1}{2 \pi i} \int_{C_{1}} \frac{1}{(\lambda-1 / 2)(\lambda+1 / 2)}(-U)[-\lambda U+I]^{-1} d \lambda \\
=\frac{1}{2 \pi i} \int_{C_{1}}-\frac{1}{(\lambda-1 / 2)(\lambda+1 / 2)} \sum_{n=0}^{\infty} \lambda^{n} U^{n+1} d \lambda \\
=\sum_{n=0}^{\infty} U^{n+1} \frac{1}{2 \pi i} \int_{-C_{1}} \frac{\lambda^{n}}{(\lambda-1 / 2)(\lambda+1 / 2)} d \lambda \\
=\sum_{n=0}^{\infty} U^{n+1}\left[(1 / 2)^{n}-(-1 / 2)^{n}\right]=\sum_{n=1}^{\infty} 4\left(\frac{U^{2}}{4}\right)^{n}=U^{2}\left(I-\frac{U^{2}}{4}\right)^{-1} \\
\frac{1}{2 \pi i} \int_{C_{2} \cdot \frac{1}{(\lambda-1 / 2)(\lambda+1 / 2)}\left(\lambda-U^{*}\right)^{-1} d \lambda}=\frac{-1}{2 \pi i} \int_{C_{2}^{\prime}} \frac{1}{\left(\frac{1}{\mu}-1 / 2\right)\left(\frac{1}{\mu}+1 / 2\right)}\left(\frac{1}{\mu}-U^{*}\right)^{-1} \frac{1}{\mu^{2}} d \mu \\
=\frac{-1}{2 \pi i} \int_{C_{2}^{\prime}} \frac{1}{\left(1-\frac{\mu}{2}\right)\left(1+\frac{\mu}{2}\right)}\left(\frac{1}{\mu}-U^{*}\right)^{-1} d \mu
\end{gathered}
$$

where $\mu=1 / \lambda$, and $C_{2}^{\prime}=\{\mu:|\mu|=1 / 2\}$.

Since the integrand is analytic in $\mu$ on $C_{2}^{\prime}$ and on the enclosed region, the above integral is equal to zero. Therefore,

$$
B=f\left(U^{*}\right)=U^{2}\left(I-U^{2} / 4\right)^{-1}
$$


and

$$
B \cdot P\left(U^{*}\right)=I+K, \quad K \in \mathcal{K}(X)
$$

by Theorem $15, \$ 2$.

A direct calculation gives

$$
K=(1 / 2 U+I)^{-1} F_{1}\left(U^{*}-1 / 2\right)-(-1 / 2 U+I)^{-1} F_{1}\left(U^{*}+1 / 2\right)
$$

where $F_{1}$ is the orthogonal projection onto $\left[e_{0}\right]$.

$\$ 4$ is devoted to proving the analogue of the Spectral Mapping Theorem for our operational calculus. We define $\hat{\sigma}_{\Phi}(A)$ as follows:

$$
\hat{\sigma}_{\boldsymbol{\Phi}}(A)= \begin{cases}\sigma_{\Phi}(A) & \text { if } A \text { is bounded } \\ \sigma_{\Phi}(A) \cup(\infty) & \text { if } A \text { is unbounded }\end{cases}
$$

We then prove the following $\Phi$-spectrum mapping theorem.

Theorem 1. If $f \in \mathbb{Q}_{\infty}^{\prime}(A)$, then $\sigma_{\Phi}[f(A)]=f\left[\hat{\sigma}_{\Phi}(A)\right]$.

We use Theorem 1 to help prove the following theorem.

Theorem 2. Let $f \in \mathbb{Q}_{\infty}^{\prime}(A)$ and $g \in \mathbb{Q}^{\prime}[f(A)]$. $\mathcal{K}(X)$.

Define $F$ by $F(\lambda)=g[f(\lambda)]$. Then $F \in \mathbb{Q}_{\infty}^{\prime}(A)$ and $F(A)=g[f(A)]+K, K \epsilon$

In $\$ 5$ we use our analytic quasi-resolvent operator to extend a theorem of Kato. In [1, Theorem 2.3], it is shown that if $\Phi_{i}(A)$ is a component of $\Phi_{A}$, then $\alpha(\lambda-A)=n$ is constant for all $\lambda \in \Phi_{i}(A)$ except for at most a countable set of points having no accumulation point in $\Phi_{i}(A)$. For these points, $\nu_{i}, i=1,2,3$, $\cdots, \alpha\left(\nu_{i}-A\right)>n$. We shall refer to this exceptional set of points as $S_{i}(A)$. In [2, Theorem 3], Kato shows that if $\lambda_{i} \in \Phi_{i}(A) \backslash S_{i}(A)$, there is a basis of $N(A-\lambda)$ consisting of $n$ vectors $x_{i}(\lambda), i=1,2, \cdots, n$, which are analytic in a neighborhood of $\lambda_{i}$. The following theorem gives a stronger result.

Theorem 3. Let $\Phi_{i}(A)$ be a component of $\Phi_{A}$, and let $\lambda_{i} \in \Phi_{i}(A) \backslash S_{i}(A)$. Let $x_{1}, x_{2}, \cdots, x_{n}$ be a basis for $N\left(A-\lambda_{i}\right)$. Then there exist vector valued functions, $x_{1}(\lambda), x_{2}(\lambda), \cdots, x_{n}(\lambda)$, analytic for all $\lambda \in \Phi_{i}(A)$ except for at most a countable set of points baving no accumulation point in $\Phi_{i}(A)$, sucb that $x_{j}\left(\lambda_{i}\right)=x_{j}, 1 \leq$ $j \leq n$, and $\left\{x_{j}(\lambda)\right\}_{j=1}^{n}$ form a basis for $N(A-\lambda)$.

In each section theorems are numbered according to the order in which they appear. The lemmas are numbered according to the theorems for which they are needed. For example, the third lemma used in proving Theorem 7 is numbered Lemma 7.3 .

2. The operational calculus. A description of the operational calculus for closed operators can be found in [6]. In this description, a complex valued 
function $f$ can be applied to the closed operator $A$ only if $f$ is analytic on a region containing $\sigma(A)$.

In this paper we will develop an operational calculus which will enlarge the set of functions which can be applied to the closed operator $A$.

Definition 1. A closed operator $A$ from a Banach space $X$ to a Banach space $Y$ is called a Fredholm operator if:

(1) the domain, $D(A)$, of $A$ is dense in $X$.

(2) $\alpha(A)=\operatorname{dim}[N(A)]<\infty$.

(3) $R(A)$, the range of $A$, is closed in $Y$.

(4) $\beta(A)$, the codimension of $R(A)$ in $Y$, is finite.

It is shown in [2, Lemma 332], that condition 4 implies condition 3. A discussion of Fredholm operators can be found in [3].

Definition 2. If $A$ is Fredholm, the index of $A, i(A)$, is defined by $i(A)=$ $\alpha(A)-\beta(A)$. We denote the set of Fredholm operators from $X$ to $X$ by $\Phi(X)$.

Definition 3. $\lambda \in \Phi_{A}$ iff $(\lambda-A) \in \Phi(X)$.

Definition 4. $\lambda \in \sigma_{\Phi}(A)$ iff $\lambda \notin \Phi_{A}$.

By [3, Theorem 2.9], $\Phi_{A}$ is open and is thus the union of a disjoint collection of connected open sets. Each such set, $\Phi_{i}(A)$, will be called a component of $\Phi_{A}$.

Theorem 1. Let $A$ be a closed operator on $X$ sucb that $\Phi_{A} \neq \varnothing$. In each component, $\Phi_{i}(A)$, of $\Phi_{A}, \alpha(\lambda-A)$ is constant for all $\lambda$ except for an isolated. set of points baving no accumulation point in $\Phi_{i}(A)$. For these exceptional sets

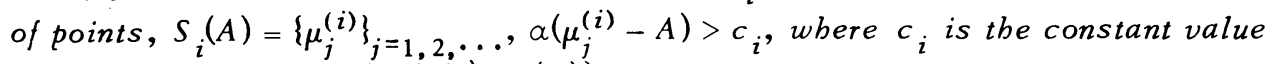
of $\alpha(\lambda-A)$ when $\lambda \in\left\{\Phi_{i}(A) \backslash S_{i}(A)\right\}$.

Proof. See [1, Theorem 2.3].

Let $A$ be a closed operator such that $\Phi_{A}$ is not empty. Let $\lambda_{i}$ be a fixed point in $\left\{\Phi_{i}(A) \backslash S_{i}(A)\right\}$. There exist subspaces $X_{i}$ and $Y_{i}$ such that

$$
\begin{array}{ll}
X=N\left(\lambda_{i}-A\right) \oplus X_{i}, & X_{i} \text { is closed, } \\
X=Y_{i} \oplus R\left(\lambda_{i}-A\right), & \operatorname{dim} Y_{i}=\beta\left(\lambda_{i}-A\right) .
\end{array}
$$

Let $F_{1 i}$ be the projection of $X$ onto $N\left(\lambda_{i}-A\right)$ along $X_{i}$, and let $F_{2 i}$ be the projection of $X$ onto $Y_{i}$ along $R\left(\lambda_{i}-A\right) . F_{1 i}$ and $F_{2 i}$ are bounded finite rank operators. $\left.\left(\lambda_{i}-A\right)\right|_{D(A) \cap X_{i}}$ has a bounded inverse, $A_{i}$,

$$
A_{i}: R\left(\lambda_{i}-A\right) \stackrel{\text { onto }}{\longrightarrow} D(A) \cap X_{i}
$$

See [3].

Let the operator $T_{i}$ be defined by: $T_{i} x=A_{i}\left(I-F_{2 i}\right) x . T_{i} \in L(X)$, where $L(X)$ denotes the set of bounded operators on $X$.

$$
\begin{aligned}
T_{i}\left(\lambda_{i}-A\right) & =I-F_{1 i} \text { on } D(A), \\
\left(\lambda_{i}-A\right) T_{i} & =I-F_{2 i} \text { on } X .
\end{aligned}
$$


Definition 5. A bounded operator $B$ will be called a right quasi-inverse of the closed operator $A$ if $R(B) \subset D(A)$ and $A B=I+K$, where $K \in \mathcal{K}(X) . K(X)$ denotes the set of all compact operators on $X$.

Definition 6. A bounded operator $B$ will be called a left quasi-inverse of the closed operator $A$ if $B A=I+K$, where $K \in \mathcal{K}(X)$.

Definition 7. An operator $B$ will be called a quasi-inverse of the closed operator $A$ if $B$ is a left and a right quasi-inverse $A$.

We see from (1.1) and (2.1) that $T_{i}$ is a quasi-inverse of $\left(\lambda_{i}-A\right)$.

Definition 8. Let $R_{\lambda}^{\prime}(A)$ be defined by $R_{\lambda}^{\prime}(A)=T_{i}\left[\left(\lambda-\lambda_{i}\right) T_{i}+I\right]^{-1}$ when $\lambda \in \Phi_{i}(A),-1 /\left(\lambda-\lambda_{i}\right) \in \rho\left(T_{i}\right)$, and $T_{i}$ is chosen as above.

Remark 1. Since $T_{i} \in L(X),-1 /\left(\lambda-\lambda_{i}\right) \in \rho\left(T_{i}\right)$ when $\lambda$ is close to $\lambda_{i}$.

Remark 2. $R_{\lambda}^{\prime}(A)$ can be chosen in different ways by making different choices of $\lambda_{i}$ and $T_{i}$. Unless stated otherwise, by $R_{\lambda}^{\prime}(A)$ we shall mean any one of these possible choices.

Theorem 2. $R_{\lambda}^{\prime}(A)$ is a quasi-inverse of $(\lambda-A)$.

Proof. $R\left[R_{i}^{\prime}(A)\right] \subset D(A)$ by the definition of $T_{i}$.

(a)

$$
(\lambda-A) T_{i}\left[\left(\lambda-\lambda_{i}\right) T_{i}+I\right]^{-1}=\left(\lambda-\lambda_{i}+\lambda_{i}-A\right) T_{i}\left[\left(\lambda-\lambda_{i}\right) T_{i}+I\right]^{-1}
$$

(b)

$$
\begin{aligned}
& \quad=\left[\left(\lambda-\lambda_{i}\right) T_{i}+I-F_{2 i}\right]\left[\left(\lambda-\lambda_{i}\right) T_{i}+I\right]^{-1}=I-F_{2 i}\left[\left(\lambda-\lambda_{i}\right) T_{i}+I\right]^{-1} \cdot \\
& T_{i}\left[\left(\lambda-\lambda_{i}\right) T_{i}+I\right]^{-1}(\lambda-A)=T_{i}\left[\left(\lambda-\lambda_{i}\right) T_{i}+I\right]^{-1}\left(\lambda-\lambda_{i}+\lambda_{i}-A\right) \\
& \quad=\left[\left(\lambda-\lambda_{i}\right) T_{i}+I\right]^{-1} T_{i}\left(\lambda-\lambda_{i}+\lambda_{i}-A\right) \\
& \quad=\left.\left[\left(\lambda-\lambda_{i}\right) T_{i}+I\right]^{-1}\left[\left(\lambda-\lambda_{i}\right) T_{i}+I-F_{1 i}\right]\right|_{D(A)} \\
& \quad=\left.\left\{I-\left[\left(\lambda-\lambda_{i}\right) T_{i}+I\right]^{-1} F_{1 i}\right\}\right|_{D(A)} \quad \text { Q.E.D. }
\end{aligned}
$$

Theorem 3. Let $L_{1}$ and $L_{2}$ be two different choices of $R_{\lambda}^{\prime}(A)$. Then $L_{1}-$ $L_{2}$ is a bounded finite rank operator.

Proof. $L_{1}(\lambda-A)=\left(I-F_{1}\right)$ and $(\lambda-A) L_{2}=I-F_{2}$, where $F_{1}$ and $F_{2}$ are bounded finite rank operators.

$$
L_{1}(\lambda-A) L_{2}=\left(I-F_{1}\right) L_{2}=L_{1}\left(I-F_{2}\right) .
$$

Therefore, $L_{1}-L_{2}=L_{1} F_{2}-F_{1} L_{2}$ which is a bounded finite rank operator. Q.E.D.

Since $T_{i}$ is bounded, $R_{\lambda}^{\prime}(A)$ is analytic in $\lambda$ when $\lambda \in \Phi_{i}(A)$ except for those points, $\nu$, such that $-1 /\left(\nu-\lambda_{i}\right) \in \sigma\left(T_{i}\right)$. We denote this set of exceptional points by $\Phi_{i}^{0}(A)$.

Theorem 4. $S_{i}(A) \subset \Phi_{i}^{0}(A)$. (See Theorem 1.)

Proof. Let $\lambda \in S_{i}(A)$. Since $\alpha(\lambda-A)>a\left(\lambda_{i}-A\right)$, there exists an $x \in X$ 
such that $x \neq 0$ and $x \in N(\lambda-A) \cap X_{i}$, where $X_{i}$ is the closed complement of $N\left(\lambda_{i}-A\right)$ chosen in the construction of $T_{i}$.

Thus

$$
\begin{aligned}
T_{i}\left(\lambda-\lambda_{i}\right) x=T_{i}(A & \left.-\lambda_{i}\right) x=-x, \text { and }\left(T_{i}+\frac{1}{\lambda-\lambda_{i}}\right) x=0 \\
& \therefore \frac{-1}{\lambda-\lambda_{i}} \in \sigma\left(T_{i}\right), \\
& \therefore \quad \lambda \in \Phi_{i}^{0}(A) .
\end{aligned}
$$

Remark 3. $\Phi_{i}^{0}(A)$ depends on the choice of $T_{i}$.

Lemma 5.1. If $\lambda \neq \lambda_{i}, \lambda \in \Phi_{A}$ if and only if $-1 /\left(\lambda-\lambda_{i}\right) \in \Phi_{T_{i}}$.

Proof.

$$
\begin{aligned}
\left(\frac{-1}{\lambda-\lambda_{i}}-T_{i}\right) & \left(\lambda-\lambda_{i}\right)\left(\lambda_{i}-A\right)=\left[-1-\left(\lambda-\lambda_{i}\right) T_{i}\right]\left(\lambda_{i}-A\right) \\
& =A-\lambda_{i}-\left(\lambda-\lambda_{i}\right)\left(I-F_{1 i}\right)=A-\lambda+\left(\lambda-\lambda_{i}\right) F_{1 i}
\end{aligned}
$$

Suppose $\lambda \in \Phi_{A}$. Then $\left[A-\lambda+\left(\lambda-\lambda_{i}\right) F_{1 i}\right] \in \Phi(X)$ by [3, Theorem 2.8]. But. $\left(\lambda_{i}-A\right) \in \Phi(X)$. Therefore,

$$
\left[\frac{-1}{\lambda-\lambda_{i}}-T_{i}\right] \in \Phi(X) \text { by [3, Theorem 3.4]. }
$$

Therefore, $-1 /\left(\lambda-\lambda_{i}\right) \in \Phi_{T_{i}}$. Conversely, suppose $-1 /\left(\lambda-\lambda_{i}\right) \in \Phi_{T_{i}}$. Then $\left(-1 /\left(\lambda-\lambda_{i}\right)-T_{i}\right)\left(\lambda-\lambda_{i}\right)\left(\lambda_{i}-A\right) \in \Phi(X)$, by [3, Theorem 2.5]. Therefore, $(A-\lambda)$ $\epsilon \Phi(X)$ by [3, Theorem 2.8]. Therefore, $\lambda \in \Phi_{A}$.

Lemma 5.2. $i(\lambda-A)$ is constant for all $\lambda$ in the same component of $\Phi_{A}$.

Proof. See [4, Chapter VII, Theorem 5.2].

Theorem 5. $\Phi_{i}^{0}(A)$ is an isolated set baving no accumulation point in $\Phi_{i}(A)$.

Proof. Let $\Phi_{i}\left(T_{i}\right)=\left\{\eta: \eta=-1 /\left(\lambda-\lambda_{i}\right)\right.$, where $\left.\lambda \in \Phi_{i}(A)\right\}$. From Lemma 5.1 we see that $\Phi_{i}\left(T_{i}\right)$ is that component of $\Phi_{T_{i}}$ which contains a neighborhood of infinity. Since $T_{i} \in L(X)$, we have $\eta \in \rho\left(T_{i}\right)$ if $\eta>\left\|T_{i}\right\|_{s \mathrm{p}}$. For these $\eta$, $a\left(\eta-T_{i}\right)=0$, and $i\left(\eta-T_{i}\right)=0$. Therefore, by Theorem 1, $a\left(\eta-T_{i}\right)=0$ for all $\eta \in \Phi_{i}\left(T_{i}\right)$, except for an isolated set which does not accumulate in $\Phi_{i}\left(T_{i}\right)$. By Lemma 5.2, $i\left(\eta-T_{i}\right)$ $=0, \forall \eta \in \Phi_{i}\left(T_{i}\right)$. Therefore, if $\eta \in \Phi_{i}\left(T_{i}\right)$ and $\alpha\left(\eta-T_{i}\right)=0, \eta$ must be in the resolvent of $T_{i}$. Therefore, $\sigma\left(T_{i}\right) \cap \Phi_{i}\left(T_{i}\right)$ is an isolated set which does not accumulate in $\Phi_{i}\left(T_{i}\right)$. Since $\Phi_{i}^{0}(A)$ is the set of all $\lambda \in \Phi_{i}(A)$ such that $-1 /\left(\lambda-\lambda_{i}\right) \in \sigma\left(T_{i}\right)$, we see that $\Phi_{i}^{0}(A)$ is an isolated set having no accumulation point in $\Phi_{i}(A)$. Q.E.D.

We have thus described a method of defining an analytic quasi-inverse, $R_{\lambda}^{\prime}(A)$, of $(\lambda-A)$ for $\lambda \in\left\{\Phi_{A} \backslash \Phi_{i}^{0}(A)\right\}$. This method can be applied to every component of $\Phi_{A}$. If we let $\Phi^{0}(A)=\bigcup_{i}^{i} \Phi_{i}^{0}(A)$, we see that $R^{\prime}(A)$ is defined for all $\lambda \in\left\{\Phi_{A} \backslash \Phi^{0}(A)\right\}$ 
Theorem 6. If the $\lambda_{i}$ used in the definition of $R_{\lambda}^{\prime}(A)$ is in $\rho(A)$, then $R_{\lambda}^{\prime}(A)=$ $(\lambda-A)^{-1}$ for all $\lambda \in\left\{\Phi_{i}(A) \backslash \Phi_{i}^{0}(A)\right\}$.

Proof. $R_{\lambda}^{\prime}(A)=T_{i}\left[\left(\lambda-\lambda_{i}\right) T_{i}+I\right]^{-1}$.

Since $\lambda_{i} \in \rho(A)$, we have that $T_{i}=\left(\lambda_{i}-A\right)^{-1}$.

$$
\begin{aligned}
(\lambda-A) & T_{i}\left[\left(\lambda-\lambda_{i}\right) T_{i}+I\right]^{-1} \\
& =\left[\left(\lambda-\lambda_{i}\right)+\left(\lambda_{i}-A\right)\right]\left(\lambda_{i}-A\right)^{-1}\left[\left(\lambda-\lambda_{i}\right)\left(\lambda_{i}-A\right)^{-1}+I\right]^{-1} \\
& =\left[\left(\lambda-\lambda_{i}\right)\left(\lambda_{i}-A\right)^{-1}+I\right]\left[\left(\lambda-\lambda_{i}\right)\left(\lambda_{i}-A\right)^{-1}+I\right]^{-1}=I .
\end{aligned}
$$

Similarly, $T_{i}\left[\left(\lambda-\lambda_{i}\right) T_{i}+I\right]^{-1}(\lambda-A)=I$. Q.E.D.

Throughout the rest of this paper, $A$ will denote a closed operator on a Banach space, $X$, with the property that $\Phi_{A} \neq \varnothing$.

If $f(\lambda)$ is a complex valued analytic function of a complex variable, we denote by $\Delta(f)$ the domain of analyticity of $f$.

Definition 9. By $\mathbb{Q}_{\infty}^{\prime}(A)$ we mean the family of all analytic functions $f(\lambda)$ with the following properties:

(1) $\sigma_{\Phi}(A) \subset \Delta(f)$,

(2) $\Delta(f)$ contains a neighborhood of $\infty$ and $f$ is analytic at $\infty$.

Definition 10. A set $D$ in the complex plane is called a Cauchy domain if the following conditions are satisfied:

(1) $D$ is open,

(2) $D$ has a finite number of components, the closures of any two of which are disjoint,

(3) the boundary of $D$ is composed of a finite positive number of closed rectifiable Jordan curves, no two of which intersect.

For a discussion of Cauchy domains, see $[6, \S 3]$.

Definition 11. Let $f \in \mathbb{Q}_{\infty}^{\prime}(A)$. The class of operators $\mathcal{F}(A)$ will be defined as follows: $B \in \mathcal{F}(A)$ if and only if

$$
B=f(\infty) I+\frac{1}{2 \pi i} \int_{+B(D)} f(\lambda) R_{\lambda}^{\prime}(A) d \lambda,
$$

where $D$ is an unbounded Cauchy domain such that

(1) $\sigma_{\Phi}(A) \subset D$

(2) $\bar{D} \subset \Delta(f)$,

(3) the boundary of $D, B(D)$, does not contain any points of $\Phi^{0}(A)$.

By $f(\infty)$ we mean $\lim _{\lambda \rightarrow \infty} f(\lambda)$. The different members of $\mathcal{F}(A)$ are obtained by making different choices of $D$ and $R_{\lambda}^{\prime}(A)$.

For the proof of the existence of a $D$ satisfying conditions 1 and 2 , see [6, Theorems 3.3 and 4.1]. Condition 3 can be satisfied because $\Phi^{0}(A)$ does not accumulate in $\Phi_{A}$.

Remark 4. All the members of $\mathcal{F}(A)$ are bounded. We proceed to show that the different members of $\mathcal{F}(A)$ differ from each other by at most a compact operator. 
Lemma 7.1. Suppose $B_{1}$ and $B_{2}$ are two members of $\mathcal{F}(A)$ obtained by using the same choice of $D$, but different choices of $R_{\lambda}^{\prime}(A)$. Then $B_{1}=B_{2}+K, K \epsilon$ $K(X)$. The compact operator mentioned in this lemma and the compact operators mentioned in the rest of the theorems of this paper turn out to be limits of finite rank operators.

Proof. By Theorem 3, the different choices of $R_{\lambda}^{\prime}(A)$ differ by at most a compact operator. The lemma follows immediately.

Lemma 7.2. Let $D$ be the unbounded Caucby domain chosen in Definition 11. Then there are at most a finite number of points of $\Phi^{0}(A)$ in $C(D)$, the comple. ment of $D$.

Proof. The lemma follows from the compactness of $C(D)$ and Theorem 5 .

Lemma 7.3. Let $D_{1}$ and $D_{2}$ be two unbounded Caucby domains satisfying the conditions in Definition 11. Then

$$
\begin{aligned}
\frac{1}{2 \pi i} \int_{+B\left(D_{1}\right)} & f(\lambda) R_{\lambda}^{\prime}(A) d \lambda-\frac{1}{2 \pi i} \int_{+B\left(D_{2}\right)} f(\lambda) R_{\lambda}^{\prime}(A) d \lambda \\
= & \frac{1}{2 \pi i} \int_{+B\left(D^{\prime}\right)} f(\lambda) R_{\lambda}^{\prime}(A) d \lambda-\frac{1}{2 \pi i} \int_{+B\left(D^{\prime \prime}\right)} f(\lambda) R_{\lambda}^{\prime}(A) d \lambda,
\end{aligned}
$$

where $D^{\prime}$ and $D^{\prime \prime}$ are bounded Cauchy domains with the following properties:

(1) $\overline{D^{\prime}}$ and $\overline{D^{\prime \prime}}$ are contained in $\Phi_{A}$,

(2) $D^{\prime}$-and $D^{\prime \prime}$ contain at most a finite number of points of $\Phi^{0}(A)$ in their interior,

(3) $D^{\prime}$ and $D^{\prime \prime}$ bave no points of $\Phi^{0}(A)$ on their boundaries.

Proof. By an argument similar to that in the proof of [6, Theorem 4.1], there exists an unbounded Cauchy domain, $D_{3}$, such that $\sigma_{\Phi}(A) \subset D_{3}$ and $\overline{D_{3}} \subset\left[D_{1} \cap D_{2}\right]$ $C \Delta(f)$. Let $D^{\prime}=D_{1}-\overline{D_{3}}$. Since $C \overline{\left(D_{3}\right)}$ contains at most a finite number of points of $\Phi^{0}(A)$ by Lemma 7.1, the same holds true for $D^{\prime}$. Since $\Phi^{0}(A)$ does not accumulate in $\Phi_{A}, D_{3}$ can be chosen so that $B\left(D_{3}\right)$ does not contain any points of $\Phi^{0}(A)$. Since $B\left(D_{1}\right)$ does not contain any points of $\Phi^{0}(A)$, we have that $B\left(D^{\prime}\right)$ does not contain any points of $\Phi^{0}(A)$. By [6, Theorem 3.1], $D^{\prime}$ is a bounded Cauchy domain and

$$
\frac{1}{2 \pi i} \int_{+B\left(D^{\prime}\right)} f(\lambda) R_{\lambda}^{\prime}(A) d \lambda=\frac{1}{2 \pi i} \int_{+B\left(D_{1}\right)} f(\lambda) R_{\lambda}^{\prime}(A) d \lambda-\frac{1}{2 \pi i} \int_{+B\left(D_{3}\right)} f(\lambda) R_{\lambda}^{\prime}(A) d \lambda \text {. }
$$

By a similar argument, there exists a bounded Cauchy domain $D^{\prime \prime}$ such that

$$
\frac{1}{2 \pi i} \int_{+B\left(D^{\prime \prime}\right)} f(\lambda) R_{\lambda}^{\prime}(A) d \lambda=\frac{1}{2 \pi i} \int_{+B\left(D_{2}\right)} f(\lambda) R_{\lambda}^{\prime}(A) d \lambda-\frac{1}{2 \pi i} \int_{+B\left(D_{3}\right)} f(\lambda) R_{\lambda}^{\prime}(A) d \lambda \text {. }
$$

Subtracting, we have

$$
\begin{aligned}
& \frac{1}{2 \pi i} \int_{+B\left(D_{1}\right)} f(\lambda) R_{\lambda}^{\prime}(A) d \lambda-\frac{1}{2 \pi i} \int_{+B\left(D_{2}\right)} f(\lambda) R_{\lambda}^{\prime}(A) d \lambda \\
&=\frac{1}{2 \pi i} \int_{+B\left(D^{\prime}\right)} f(\lambda) R_{\lambda}^{\prime}(A) d \lambda-\frac{1}{2 \pi i} \int_{+B(D)} f(\lambda) R_{\lambda}^{\prime}(A) d \lambda . \quad \text { Q.E.D. }
\end{aligned}
$$


Lemma 7.4. Let $\mu_{i} \in \Phi_{i}^{0}(A)$. Let $D$ be a bounded Cauchy domain with the following properties:

(1) $\bar{D} \subset \Phi_{i}(A)$,

(2) $\mu_{i} \in D$, Then

(3) no other points of $\Phi^{0}(A)$ are contained in $\bar{D}$.

$$
\frac{1}{2 \pi i} \int_{+B(D)} R_{\lambda}^{\prime}(A) d \lambda=K, \quad K \in K(X) .
$$

Proof. $R_{\lambda}^{\prime}(A)=T_{i}\left[\left(\lambda-\lambda_{i}\right) T_{i}+I\right]^{-1}$.

If $\lambda_{i} \in \bar{D}, D$ can be replaced by a bounded Cauchy domain, $D_{1}$, such that $\lambda_{i} \notin \bar{D}_{1}$ and

$$
\frac{1}{2 \pi i} \int_{+B(D)} R_{\lambda}^{\prime}(A) d \lambda=\frac{1}{2 \pi i} \int_{+B\left(D_{1}\right)} R_{\lambda}^{\prime}(A) d \lambda \text {. }
$$

This replacement is possible because of the properties of $D$ and the analyticity of $R_{\lambda}^{\prime}(A)$. Therefore, we shall assume that $\lambda_{i} \notin \bar{D}$.

$$
\begin{aligned}
\int_{+B(D)} R_{\lambda}^{\prime}(A) d \lambda & =\int_{+B(D)} T_{i}\left[\left(\lambda-\lambda_{i}\right) T_{i}+I\right]^{-1} d \lambda \\
& =\int_{+B(D)} \frac{1}{\lambda-\lambda_{i}} T_{i}\left(T_{i}+\frac{1}{\lambda-\lambda_{i}}\right)^{-1} d \lambda .
\end{aligned}
$$

Let $\mu=1 /\left(\lambda-\lambda_{i}\right)$,

$$
\int_{+B(D)} R_{\lambda}^{\prime}(A) d \lambda=\int_{\Gamma} \frac{-1}{\mu} T_{i}\left(T_{i}+\mu\right)^{-1} d \mu
$$

with $\Gamma$ being the oriented image of $+B(D)$ under the mapping $\mu=1 /\left(\lambda-\lambda_{i}\right)$.

$$
\begin{gathered}
T_{i}=\left(\mu+T_{i}\right)-\mu, \quad T_{i}\left(T_{i}+\mu\right)^{-1}=I-\mu\left(T_{i}+\mu\right)^{-1} ; \\
\therefore \int_{+B(D)} R_{\lambda}^{\prime}(A) d \lambda=\int_{\Gamma} \frac{-1}{\mu} I d \mu+\int_{\Gamma}\left(T_{i}+\mu\right)^{-1} d \mu .
\end{gathered}
$$

Since $\lambda_{i} \notin \bar{D}$ and $D$ is bounded, $\dot{\Gamma}$ is bounded and $0 \notin \dot{\Gamma} \cup \Gamma$ where $\dot{\Gamma}$ is the interior of $\Gamma$.

$$
\begin{aligned}
& \cdot \int_{\Gamma} \frac{-1}{\mu} I d \mu=0 . \\
& \int_{\Gamma}\left(T_{i}+\mu\right)^{-1} d \mu=\int_{\Gamma}\left[\mu-\left(-T_{i}\right)\right]^{-1} d \mu .
\end{aligned}
$$

Since $\mu_{i} \in \bar{D}, 1 /\left(\mu_{i}-\lambda_{i}\right) \in \dot{\Gamma} .-1 /\left(\mu_{i}-\lambda_{i}\right) \in \sigma\left(T_{i}\right)$ because $\mu_{i} \in \Phi_{i}^{0}(A)$. Further, since $\mu_{i} \in \Phi_{A},-1 /\left(\mu_{i}-\lambda_{i}\right) \in \Phi_{T_{i}}$ by Lemma 5.1. Therefore, $1 /\left(\mu_{i}-\lambda_{i}\right) \epsilon$ $o\left(-T_{i}\right) \cap \Phi_{\left(-T_{i}\right)}$. There are no other points of $\sigma\left(-T_{i}\right)$ in $\dot{\Gamma}$. Therefore, by [4, Chapter VI, Theorem $4.5^{\circ}$ and the proof of Theorem 4.2], we have that $\int_{\Gamma}\left[\mu-\left(-T_{i}\right)\right]^{-1} d \mu$ is a bounded finite rank operator. Therefore,

$$
\frac{1}{2 \pi i} \int_{+B(D)} R_{\lambda}^{\prime}(A) d \lambda=K, \quad K \in \mathcal{K}(X) .
$$

Lemma 7.5. Let $\lambda$ and $\mu$ be in $\Phi_{A}$. Then

$$
R_{\lambda}^{\prime}(A) \cdot R_{\mu}^{\prime}(A)=\frac{R_{\lambda}^{\prime}(A)-R_{\mu}^{\prime}(A)}{\mu-\lambda}+K, \quad K \in \mathcal{K}(X) .
$$


Proof.

$$
\begin{aligned}
R_{\lambda}^{\prime}(A)(\lambda-A)\left[R_{\lambda}^{\prime}(A)-R_{\mu}^{\prime}(A)\right](\mu- & A) R_{\mu}^{\prime}(A) \\
& =\left(I-F_{1}\right)\left[P_{\lambda}^{\prime}\left((A)-R_{\mu}^{\prime}(A)\right]\left(I-F_{2}\right), \quad F_{1}, F_{2} \in \mathcal{K}(X),\right. \\
& =R_{\lambda}^{\prime}(A)-R_{\mu}^{\prime}(A)+F, \quad F \in K(X) .
\end{aligned}
$$

We also have that

$$
\begin{array}{r}
R_{\lambda}^{\prime}(A)(\lambda-A)\left[R^{\prime}(A)-R_{\mu}^{\prime}(A)\right](\mu-A) R_{\mu}^{\prime}(A) \\
=R_{\lambda}^{\prime}(A)\left(I-F_{3}\right)(\mu-A) R_{\mu}^{\prime}(A)-R_{\lambda}^{\prime}(A)(\lambda-A)\left(I-F_{4}\right) R_{\mu}^{\prime}(A) \\
=(\mu-\lambda) R_{\lambda}^{\prime}(A) R_{\mu}^{\prime}(A)-R_{\lambda}^{\prime}(A) F_{3}\left(I-F_{2}\right)+\left(I-F_{1}\right) F_{4} R_{\mu}^{\prime}(A) \\
=(\mu-\lambda) R_{\lambda}^{\prime}(A) R_{\mu}^{\prime}(A)+F_{0}, \quad F_{0} \in K(X) ; \\
. \quad R_{\lambda}^{\prime}(A)-R_{\mu}^{\prime}(A)+F=(\mu-\lambda) R_{\lambda}^{\prime}(A) R_{\mu}^{\prime}(A)+F_{0} \\
. \quad R_{\lambda}^{\prime}(A) R_{\mu}^{\prime}(A)=\frac{R_{\lambda}^{\prime}(A)-R_{\mu}^{\prime}(A)}{\mu-\lambda}+K, \quad K \in \mathbb{K}(X) .
\end{array}
$$

Note. $K$ depends analytically on $\mu$ and $\lambda$.

Lemma 7.6. Let $\mu_{i}$ and $D$ be as in Lemma 7.4. Let $f(\lambda)$ and $g(\lambda)$ be such that $\bar{D} \subset[\Delta(f) \cap \Delta(g)]$. Then

$$
\begin{aligned}
\frac{1}{2 \pi i} \int_{+B(D)} f(\lambda) R_{\lambda}^{\prime}(A) d \lambda \cdot \frac{1}{2 \pi i} \int_{+B(D)} g(\lambda) R_{\lambda}^{\prime}(A) d \lambda & \\
= & \frac{1}{2 \pi i} \int_{+B(D)} f(\lambda) g(\lambda) R_{\lambda}^{\prime}(A) d \lambda+K, \quad K \in K(X) .
\end{aligned}
$$

Proof. By [6, Theorem 3.3], there exists a bounded Cauchy domain, $D_{1}$, such that $\mu_{i} \in D_{1}$ and $\overline{D_{1}} \subset D \subset[\Lambda(f) \cap \Delta(g)]$.

$$
\int_{+B(D)} f(\lambda) R_{\lambda}^{\prime}(A) d \lambda=\int_{+B\left(D_{1}\right)} f(\mu) R_{\mu}^{\prime}(\Lambda) d \mu ;
$$

$$
\begin{aligned}
& \therefore \frac{1}{2 \pi i} \int_{+B(D)} f(\lambda) R_{\lambda}^{\prime}(A) d \lambda \cdot \frac{1}{2 \pi i} \int_{+B(D)} g(\lambda) R_{\lambda}^{\prime}(A) d \lambda \\
&=\frac{1}{2 \pi i} \int_{+B\left(D_{1}\right)} f(\mu) R_{\mu}^{\prime}(A) d \mu \cdot \frac{1}{2 \pi i} \int_{+B(D)} g(\lambda) R_{\lambda}^{\prime}(A) d \lambda \\
&=\frac{-1}{4 \pi^{2}} \int_{+B\left(D_{1}\right)} \int_{+B(D)} f(\mu) g(\lambda) R_{\mu}^{\prime}(A) R_{\lambda}^{\prime}(A) d \lambda d \mu \\
&=\frac{-1}{4 \pi^{2}} \int_{+B\left(D_{1}\right)} \int_{+B(D)} f(\mu) g(\lambda)\left(\frac{R_{\lambda}^{\prime}(A)-R_{\mu}^{\prime}(A)}{\mu-\lambda}\right) d \lambda d \mu \\
&-\frac{1}{4 \pi^{2}} \int_{+B\left(D_{1}\right)} \int_{+B(D)} f(\mu) g(\lambda) K(\mu, \lambda) d \lambda d \mu, \quad K(\mu, \lambda) \in K(X),
\end{aligned}
$$

by Lemma 7.5 . 
Since $K(\mu, \lambda)$ is a compact operator, the last integral is a compact operator.

$$
\begin{aligned}
\frac{-1}{4 \pi^{2}} \int_{+B\left(D_{1}\right)} \int_{+B(D)} & f(\mu) g(\lambda)\left(\frac{R_{\lambda}^{\prime}(A)-R_{\mu}^{\prime}(A)}{\mu-\lambda}\right) d \lambda d \mu \\
= & \frac{1}{2 \pi i} \int_{+B(D)} g(\lambda) R_{\lambda}^{\prime}(A) \frac{1}{2 \pi i} \int_{+B\left(D_{1}\right)} \frac{f(\mu)}{\mu-\lambda} d \mu d \lambda \\
& -\frac{1}{2 \pi i} \int_{+B\left(D_{1}\right)} f(\mu) R_{\mu}^{\prime}(A) \frac{1}{2 \pi i} \int_{+B(D)} \frac{g(\lambda)}{\mu-\lambda} d \lambda d \mu .
\end{aligned}
$$

Since $\lambda$ is not contained in $\overline{D_{1}}$, the first of the above integrals equals zero.

$$
\frac{1}{2 \pi i} \int_{+B(D)} \frac{g(\lambda)}{\mu-\lambda} d \lambda=-g(\mu)
$$

Therefore, the last integral is equal to

$$
\frac{1}{2 \pi i} \int_{+B\left(D_{1}\right)} f(\mu) g(\mu) R_{\mu}^{\prime}(A) d \mu=\frac{1}{2 \pi i} \int_{+B(D)} f(\lambda) g(\lambda) R_{\lambda}^{\prime}(A) d \lambda \text {. Q.E.D. }
$$

Lemma 7.7. Let $\mu_{i}$ and $D$ be as in Lemma 7.4. Let $f(\lambda)$ be sucb that $\bar{D} \subset$ $\Delta(f)$. Then,

$$
\frac{1}{2 \pi i} \int_{+B(D)} f(\lambda) R_{\lambda}^{\prime}(A) d \lambda=K, \quad K \in K(X)
$$

Proof. By Lemma 7.6,

$$
\begin{array}{r}
\frac{1}{2 \pi i} \int_{+B(D)} f(\lambda) R_{\lambda}^{\prime}(A) d \lambda=\frac{1}{2 \pi i} \int_{+B(D)} f(\lambda) R_{\lambda}^{\prime}(A) d \lambda \frac{1}{2 \pi i} \int_{+B(D)} R_{\lambda}^{\prime}(A) d \lambda+K, \\
K \in K(X) .
\end{array}
$$

By Lemma 7.4, $(1 / 2 \pi i) \int_{+B(D)} R_{\lambda}^{\prime}(A) d \lambda=K_{1}, K_{1} \in \mathcal{K}(X)$. Therefore,

$$
\frac{1}{2 \pi i} \int_{+B(D)} f(\lambda) R_{\lambda}^{\prime}(A) d \lambda=K_{2}, \quad K_{2} \in K(X) .
$$

Lemma 7.8. Let $f \in \mathbb{P}_{\infty}^{\prime}(A)$, and let $D_{1}$ and $D_{2}$ be unbounded Cauchy domains such that $\sigma_{\Phi}(A) \subset\left[D_{1} \cap D_{2}\right],\left[\overline{D_{1}} \cup \overline{D_{2}}\right] \subset \Delta(f)$, and $\left[B\left(D_{1}\right) \cup B\left(D_{2}\right)\right]$ does not contain points of $\Phi^{0}(A)$. Then

$$
\frac{1}{2 \pi i} \int_{+B\left(D_{1}\right)} f(\lambda) R_{\lambda}^{\prime}(A) d \lambda-\frac{1}{2 \pi i} \int_{+B\left(D_{2}\right)} f(\lambda) R_{\lambda}^{\prime}(A) d \lambda=K, \quad K \in K(X)
$$

Proof. By Lemna 7.3,

$$
\begin{aligned}
\frac{1}{2 \pi i} \int_{+B\left(\cap_{1}\right)} f(\lambda) R_{\lambda}^{\prime}(A) d \lambda-\frac{1}{2 \pi i} \int_{+B\left(D_{2}\right)} f(\lambda) R_{\lambda}^{\prime}(A) d \lambda \\
=\frac{1}{2 \pi i} \int_{+B\left(D^{\prime}\right)} f(\lambda) R_{\lambda}^{\prime}(A) d \lambda-\frac{1}{2 \pi i} \int_{+B\left(D^{\prime \prime}\right)} f(\lambda) R_{\lambda}^{\prime}(A) d \lambda
\end{aligned}
$$

where $D^{\prime}$ and $D^{\prime \prime}$ have the properties described in Lemma 7.3. In particular, $D^{\prime}$ has at most a finite number of points of $\Phi^{0}(A)$ in its interior. Therefore, $(1 / 2 \pi i) \int_{+B\left(D^{\prime}\right)} f(\lambda) R_{\lambda}^{\prime}(A) d \lambda$ is a finite sum of integrals of the form $(1 / 2 \pi i) \int_{+B(D)} f(\lambda) R_{\lambda}^{\prime}(A) d \lambda$, where $D$ is as in Lemma 7.4. By Lemma 7.7, each of these 
integrals is a compact operator. Therefore, $(1 / 2 \pi i) \int_{+B\left(D^{\prime}\right)} f(\lambda) R_{\lambda}^{\prime \prime}(A) d \lambda$ is a compact operator. Similarly, $\int_{+B\left(D^{\prime \prime}\right)} f(\lambda) R_{\lambda}^{\prime}(A) d \lambda$ is a compact operator. Therefore,

$$
\frac{1}{2 \pi i} \int_{+B\left(D_{1}\right)} f(\lambda) R_{\lambda}^{\prime}(A) d \lambda-\frac{1}{2 \pi i} \int_{+B\left(D_{2}\right)} f(\lambda) R_{\lambda}^{\prime}(A) d \lambda=K, \quad K \in K(X) . \quad \text { Q.E.D. }
$$

Theorem 7. Let $B_{1}, B_{2} \in \mathcal{F}(A)$. Then $B_{1}-B_{2}=K, K \in \mathcal{K}(X)$.

Proof. The theorem follows immediately from Lemma 7.1 and Lemma 7.8.

Definition 12. Let $f \in \mathbb{Q}_{\infty}^{\prime}(A)$. By $f(A)$ we mean an arbitrary operator in the set $\mathcal{F}(A)$.

From Theorem 7 we have that two different choices of $f(A)$ can differ by at most a compact operator.

Theorem 8. Let $f(\lambda)$ and $g(\lambda)$ be in $\mathbb{Q}_{\infty}^{\prime}(A)$. Let $\alpha$ and $\beta$ be complex num. bers. If we use the same $R_{\lambda}^{\prime}(A)$ and we integrate along the same curve, then $\alpha f(A)+\beta g(A)=(\alpha f+\beta g)(A)$, where $(\alpha f+\beta g)$ is defined by $(\alpha f+\beta g) \lambda=\alpha f(\lambda)+$ $\beta g(\lambda)$. If we use different operators for $R_{\lambda}^{\prime}(A)$ or if we integrate about different regions, then $\alpha f(A)+\beta g(A)=(\alpha f+\beta g)(A)+K$, where $K \in \mathcal{K}(X)$.

Proof. The first part of the theorem is obvious and the second part follows from Theorem 7 .

Theorem 9. Let $f(\lambda)$ and $g(\lambda)$ be in $\mathbb{Q}_{\infty}^{\prime}(A)$. Then $f(A) \cdot g(A)=(f \cdot g)(A)+K$, $K \in \mathcal{K}(X)$.

$(f \cdot g)$ is defined by $(f \cdot g)(\lambda)=f(\lambda) \cdot g(\lambda)$.

Once the curve of integration is chosen and the choice of $R_{\lambda}^{\prime}(A)$ is made, the compact operator, $K$, of this theorem can be computed directly.

Proof. By [6, Theorem 3.3], there exist unbounded Cauchy domains $D_{1}$ and $D_{2}$ such that $\sigma_{\Phi}(A) \subset D_{1}, \overline{D_{1}} \subset D_{2} \subset[\Delta(f) \cap \Delta(g)]$. Further, since $C \overline{\left(D_{1}\right)}$ contains at most a finite number of points of $\Phi^{0}(A), D_{1}$ and $D_{2}$ can be chosen so that $\left(D_{2}-\overline{D_{1}}\right)$ does not contain any points of $\Phi^{0}(A)$.

$$
\begin{aligned}
f(A)= & f(\infty) I+\frac{1}{2 \pi i} \int_{+B\left(D_{1}\right)} f(\mu) R_{\mu}^{\prime}(A) d \mu, \\
g(A)= & g(\infty) I+\frac{1}{2 \pi i} \int_{+B\left(D_{2}\right)} g(\lambda) R_{\lambda}^{\prime}(A) d \lambda, \\
f(A) \cdot g(A)= & f(\infty) g(\infty) I+f(\infty) \frac{1}{2 \pi i} \int_{+B\left(D_{2}\right)} g(\lambda) R_{\lambda}^{\prime}(A) d \lambda \\
& +g(\infty) \frac{1}{2 \pi i} \int_{+B\left(D_{1}\right)} f(\mu) R_{\mu}^{\prime}(A) d \mu \\
& +\frac{-1}{4 \pi^{2}} \int_{+B\left(D_{2}\right)} \int_{+B\left(D_{1}\right)} f(\mu) g(\lambda) R_{\mu}^{\prime}(A) R_{\lambda}^{\prime}(A) d \mu d \lambda .
\end{aligned}
$$

As in the proof of Lemma 7.6, the last integral is equal to 


$$
\begin{aligned}
\frac{1}{2 \pi i} \int_{+B\left(D_{1}\right)} f(\mu) R_{\mu}^{\prime}(A) & \frac{1}{2 \pi i} \int_{+B\left(D_{2}\right)} \frac{g(\lambda)}{\lambda-\mu} d \lambda d \mu \\
& -\frac{1}{2 \pi i} \int_{+B\left(D_{2}\right)} g(\lambda) R_{\lambda}^{\prime}(A) \frac{1}{2 \pi i} \int_{+B\left(D_{1}\right)} \frac{f(\mu)}{\mu-\lambda} d \mu d \lambda+K, \quad K \in K(X) .
\end{aligned}
$$

Since $\mu \in D_{2}, \int_{+B\left(D_{2}\right)} g(\lambda) /(\lambda-\mu) d \lambda=g(\mu)-g(\infty)$. See [6, proof of Theorem 4.3].

Therefore,

$$
\begin{aligned}
\frac{1}{2 \pi i} \int_{+B\left(D_{1}\right)} f(\mu) & R_{\mu}^{\prime}(A) \frac{1}{2 \pi i} \int_{+B\left(D_{2}\right)} \frac{g(\lambda)}{\lambda-\mu} d \lambda d \mu \\
& =\frac{1}{2 \pi i} \int_{+B\left(D_{1}\right)} f(\mu) R_{\mu}^{\prime}(A)[g(\mu)-g(\infty)] d \mu \\
& =\frac{1}{2 \pi i} \int_{+B\left(D_{1}\right)} f(\mu) g(\mu) R_{\mu}^{\prime}(A) d \mu-g(\infty) \frac{1}{2 \pi i} \int_{+B\left(D_{1}\right)} f(\mu) R_{\mu}^{\prime}(A) d \mu .
\end{aligned}
$$

Since $\lambda \notin D_{1},(1 / 2 \pi i) \int_{+B\left(D_{1}\right)} f(\mu) /(\mu-\lambda) d \mu=f(\infty)$. See [6, proof of Theorem 4.3]. Therefore,

$$
\frac{1}{2 \pi i} \int_{+B\left(D_{2}\right)} g(\lambda) R_{\lambda}^{\prime}(A) \frac{1}{2 \pi i} \int_{+B\left(D_{1}\right)} \frac{f(\mu)}{\mu-\lambda} d \mu d \lambda=f(\infty) \frac{1}{2 \pi i} \int_{+B\left(D_{2}\right)} g(\lambda) R_{\lambda}^{\prime}(A) d \lambda
$$

Therefore,

$$
\begin{aligned}
f(A) \cdot g(A)=f(\infty) \cdot g(\infty) I & +\frac{1}{2 \pi i} \int_{+B\left(D_{1}\right)} f(\mu) g(\mu) R_{\mu}^{\prime}(A) d \mu \\
& =(f \cdot g)(A)+K, \quad K \in K(X) . \quad \text { Q.E.D. }
\end{aligned}
$$

Theorem 10. Let $f(\lambda) \equiv 1$. Then $f(A)=I+K, K \in \mathcal{K}(X)$.

Proof. $f(A)=f(\infty) I+(1 / 2 \pi i) \int_{+B(D)} R_{\lambda}^{\prime}(A) d \lambda, f(\infty) I=I$. As in [6, Theorem 4.2],

$$
\frac{1}{2 \pi i} \int_{+B(D)} R_{\lambda}^{\prime}(A) d \lambda=\frac{-1}{2 \pi i} \int_{+B\left(D_{1}\right)} R_{\lambda}^{\prime}(A) d \lambda
$$

where $D_{1}=C(\bar{D})$ is a bounded Cauchy domain. $D_{1} \subset \Phi_{A}$. By Lemma 7.2, $D_{1}$ contains at most a finite number of points of $\Phi^{0}(A)$ in its interior. Therefore, by Lemma $7.4,(1 / 2 \pi i) \int_{+B\left(D_{2}\right)} R_{\lambda}^{\prime}(A) d \lambda$ is the sum of a finite number of compact operators. Therefore, $(1 / 2 \pi i) \int_{+B\left(D_{1}\right)} R_{\lambda}^{\prime}(A) d \lambda$ is compact. Therefore,

$$
f(A)=I+K, \quad K \in \mathcal{K}(X) \text {. Q.E.D. }
$$

Theorem 11. Let $A$ be a bounded operator on $X$, and let $f \in \mathbb{Q}_{\infty}^{\prime}(A)$. Then $f(A)=(1 / 2 \pi i) \int_{+\underline{B}(D)} f(\lambda) R_{\lambda}^{\prime}(A) d \lambda$, where $D$ is a bounded Cauchy domain such that $\sigma_{\Phi}(A) \subset D$, and $\bar{D} \subset \Delta(f)$.

Proof. Let $C$ be a circle of radius $r$, so chosen that $D$ lies in the interior of $C, C$ and the region exterior to it lie in $\Delta(f)$, and $r$ is greater than the spectral radius of $A$.

By Theorem $6, R_{\lambda}^{\prime}(A)=(\lambda-A)^{-1}$ in that component of $\Phi_{A}$ containing $C$ and 
the region exterior to it. The rest of the proof is entirely analogous to that of $[6$, Theorem 4.4].

Definition 13. Let $A \in L(X)$. By $\mathbb{Q}^{\prime}(A)$ we mean the family of all analytic

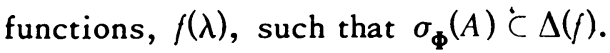

Definition 14. Let $f \in \mathbb{Q}^{\prime}(A)$. The class of operators $\mathcal{F}^{*}(A)$ will be defined as follows: $B \in \mathcal{F}^{*}(A)$ if and only if

$$
B=\frac{1}{2 \pi i} \int_{+B(D)} f(\lambda) R_{\lambda}^{\prime}(A) d \lambda
$$

where $D$ is a bounded Cauchy domain such that

(1) $\sigma_{\Phi}(A) \subset D$,

(2) $\bar{D} \subset \Delta(f)$,

(3) $B(D)$ does not contain any point of $\Phi^{0}(A)$.

Definition 15. Let $f \in \mathbb{Q}^{\prime}(A)$. By $f^{*}(A)$ we mean an arbitrary operator in $\mathcal{F}^{*}(A)$.

Theorem 12. Let $B_{1}, B_{2} \in \mathcal{F}^{*}(A)$. Then $B_{1}-B_{2}=K, K \in \mathcal{K}(X)$.

Proof. The proof is entirely analogous to the proof of Theorem 7.

Theorem 12 shows that two different choices of $f^{*}(A)$ can differ by at most a compact operator.

Theorem 11 shows that if $f \in \mathbb{Q}_{\infty}^{\prime}(A)$, where $A \in L(X)$, then $f^{*}(A)=f(A)$.

Theorems 8 and 9 have obvious analogues to $f^{*}(A)$ and the proofs are completely similar.

Theorem 13. Let $A \in L(X)$, and let $f(\lambda) \equiv 1$. Then $f^{*}(A) \equiv I+K, K \in \mathcal{K}(X)$.

Proof. $f^{*}(A)=\int_{+B(D)} R_{\lambda}^{\prime}(A) d \lambda$. Combining Theorems 10 and 11 , the theorem follows immediately.

Theorem 14. Let $A \in L(X)$. Let $f(\lambda)=\lambda$. Then $f^{*}(A)=A+K, K \in \mathcal{K}(X)$.

Proof. Since $A \in L(X), \sigma(A)$ is bounded. Since $f(\lambda)$ is entire, we can choose $D$ to be the interior of a circle whose radius, $r$, is greater than the spectral radius of $A$. Then, by Theorem $6, R_{\lambda}^{\prime}(A)=(\lambda-A)^{-1}$ along $B(D)$. The rest of the proof is then the same as that for the standard operational calculus. See [4, Chapter VI, Theorem 3.3].

Theorem 15. Let $A$ be closed and let $f \in \mathbb{Q}_{\infty}^{\prime}(A)$. Suppose $f(\lambda)$ bas no zeros on $\sigma_{\Phi}(A)$ or at $\infty$. Then $f(A)$ bas a quasi-inverse in $L(X)$.

Proof. Let $g(\lambda)=1 / f(\lambda)$, whenever $f(\lambda) \neq 0 . \Delta(g)$ contains $\sigma_{\Phi}(A)$ and a neighborhood of $\infty$. Therefore, $g \in \mathbb{Q}_{\infty}^{\prime}(A)$.

By Theorems 9 and 10,

$$
f(A) g(A)=I+K_{1}, \quad g(A) f(A)=I+K_{2}, \quad K_{1}, K_{2} \in K(X) . \quad \text { Q.E.D. }
$$


Theorem 16. Let $A$ be bounded and let $f \in \mathbb{Q}^{\prime}(A)$. Suppose $f(\lambda)$ bas no zeros on $\sigma_{\Phi}(A)$. Then $f^{*}(A)$ bas a quasi-inverse in $L(X)$.

Proof. The proof is analogous to that of Theorem 15.

3. Polynomials. If $f(\lambda)$ is a polynomial function, and $A \in L(X)$, then $f \epsilon$ $\mathbb{Q}^{\prime}(A)$, and the operational calculus which has been developed applies to $f^{*}(A)$.

However, if $A$ is unbounded, the operational calculus described in $\$ 2$ does not apply to $A$ because $f \notin \mathbb{Q}_{\infty}^{\prime}(A)$. In this section we will develop an operational calculus for polynomials.

Lemma 1.1. Let $f(\lambda)=(\alpha-\lambda)^{-1}, \alpha \in\left[\Phi_{A} \backslash \Phi^{0}(A)\right]$. Then $\exists K \in \mathcal{K}(X)$ such that $f(A)-K=R_{\alpha}^{\prime}(A)$. $\S 2$

Proof. $f(A)=f(\infty) I+(1 / 2 \pi i) \int_{+B(D)}(\alpha-\lambda)^{-1} R_{\lambda}^{\prime}(A) d \lambda, f(\infty)=0$. By Lemma 7.5.

$$
\begin{aligned}
& R^{\prime}(A)= R_{\alpha}^{\prime}(A)+(\alpha-\lambda) R_{\lambda}^{\prime}(A) R_{\alpha}^{\prime}(A)+K(\alpha, \lambda), \quad K(\alpha, \lambda) \in K(X) . \\
& . \quad f(A)= \frac{1}{2 \pi i} \int_{+B(D)} \frac{1}{\alpha-\lambda} R_{\alpha}^{\prime}(A) d \lambda+\frac{1}{2 \pi i} \int_{+B(D)} R_{\lambda}^{\prime}(A) R_{\alpha}^{\prime}(A) d \lambda \\
&+\frac{1}{2 \pi i} \int_{+B(D)} \frac{1}{\alpha-\lambda} K(\alpha, \lambda) d \lambda, \\
& \frac{1}{2 \pi i} \int_{+B(D)} R_{\lambda}^{\prime}(A) R_{\alpha}^{\prime}(A) d \lambda=\frac{1}{2 \pi i} \int_{+B(D)} R_{\lambda}^{\prime}(A) d \lambda R_{\alpha}^{\prime}(\lambda) .
\end{aligned}
$$

$(1 / 2 \pi i) \int_{+B(D ;} ;_{\lambda}^{\prime}(A) d \lambda$ is a compact operator as shown in the proof of Theorem 10, $\$ 2$. Therefore, $(1 / 2 \pi i) \int_{+B(D)} R_{\lambda}^{\prime}(A) R_{a}^{\prime}(A) d \lambda$ is compact.

$(1 / 2 \pi i) \int_{+B(D)}(\alpha-\lambda)^{-1} K(\alpha, \lambda) d \lambda$ is compact, because $K(\alpha, \lambda)$ is compact for all $\lambda \in B(D)$.

$$
\frac{1}{2 \pi i} \int_{+B(D)} \frac{1}{\alpha-\lambda} R_{\alpha}^{\prime}(A) d \lambda=\frac{1}{2 \pi i} \int_{+B\left(D_{1}\right)} \frac{-1}{\alpha-\lambda} R_{\alpha}^{\prime}(A) d \lambda=R_{\alpha}^{\prime}(A)
$$

where $D_{1}=C(\bar{D})$. Therefore, $f(A)=R_{\alpha}^{\prime}(A)+K, K \in \mathcal{K}(X)$. Q.E.D.

Definition 1. Suppose $P(\lambda)=\sum_{i=0}^{n} a_{i} \lambda^{i}$. By $P(A)$ we mean $\sum_{i=0}^{n} a_{i} A^{i}$.

If $P(\lambda)$ is a polynomial of degree $n$, clearly the domain of $P(A), D[P(A)]$, is equal to $D\left(A^{n}\right)$.

Lemma 1.2. Let $n$ be a positive integer. We can choose $R_{\lambda}^{\prime}(A)$ so that $\forall j \leq$ $n$ the following bold:

(1) $R\left\{\left[R_{\lambda}^{\prime}(A)\right]^{j}\right\} \subset D\left(A^{j}\right)$,

(2) $(\lambda-A)^{j}\left[R_{\lambda}^{\prime}(A)\right]^{j}=I+F_{j}$, where $F_{j}$ is a bounded finite rank operator.

Proof. Let $\lambda_{i} \in\left\{\Phi_{i}(A) \backslash S_{i}(A)\right\}$, where $S_{i}(A)$ is as in Theorem $1, \S 2$. $\left(\lambda_{i}-A\right)$ $\epsilon \Phi(X)$. By [3, Theorem 2.5], $D\left(A^{n}\right)=D\left[\left(\lambda_{i}-A\right)^{n}\right]$ is dense in $X$. We can set $X=R\left(\lambda_{i}-A\right) \oplus Y_{i}$, where $Y_{i}$ is a finite dimensional subspace of $X$ contained in $\left.D\left[\lambda_{i}-A\right)^{n}\right]$. The last requirement can be satisfied as seen in the proof of [3, Theorem 2.5]. Therefore, the $T_{i}$ used in the definition of $R_{\lambda}^{\prime}(A)$ for 
$\lambda \in \Phi_{i}(A)$, can be chosen so that $\left(\lambda_{i}-A\right) T_{i}=I-F_{2 i}$, with $F_{2 i}$ being the projection of $X$ onto $Y_{i}$ along $R\left(\lambda_{i}-A\right)$.

Claim. $(\lambda-A)^{j}\left[R_{\lambda}^{\prime}(A)\right]^{j}$ is defined and is equal to

$$
I-\sum_{\nu=0}^{j-1}(\lambda-A)^{\nu} F_{2 i}\left[\left(\lambda-\lambda_{i}\right) T_{i}+I\right]^{-1}\left[R_{\lambda}^{\prime}(A)\right]^{\nu}
$$

when $j \leq n$.

Proof of claim. The proof proceeds by induction. If $j=1,(\lambda-A) R_{\lambda}^{\prime}(A)=$ $(\lambda-A) T_{i}\left[\left(\lambda-\lambda_{i}\right) T_{i}+I\right]^{-1}=I-F_{2 i}\left[\left(\lambda-\lambda_{i}\right) T_{i}+I\right]^{-1}$. Suppose the claim is true for $j-1$. Then

$$
(\lambda-A)^{j-1}\left[R_{\lambda}^{\prime}(A)\right]^{j-1}=I-\sum_{\nu=0}^{j-2}(\lambda-A)^{\nu} F_{2 i}\left[\left(\lambda-\lambda_{i}\right) T_{i}+I\right]^{-1}\left[R_{\lambda}^{\prime}(A)\right]^{\nu} .
$$

Then

$(\lambda-A)^{j}\left[R_{\lambda}^{\prime}(A)\right]^{j}=I-F_{2 i}\left[\left(\lambda-\lambda_{i}\right) T_{i}+I\right]^{-1}-\sum_{\nu=1}^{j-1}(\lambda-A)^{\nu} F_{2 i}\left[\left(\lambda-\lambda_{i}\right) T_{i}+I\right]^{-1}\left[R_{\lambda}^{\prime}(A)\right]^{\nu}$.

The expression in the summation is defined because $R\left(F_{2 i}\right) \subset D\left(A^{n}\right)$. Combining terms we get

$$
(\lambda-A)^{j}\left[R_{\lambda}^{\prime}(A)\right]^{j}=I-\sum_{\nu=0}^{j-1}(\lambda-A)^{\nu} F_{2 i}\left[\left(\lambda-\lambda_{i}\right) T_{i}+I\right]^{-1}\left[R_{\lambda}^{\prime}(A)\right]^{\nu} .
$$

The claim is now proven by inductiun. Since $F_{2 i}$ is a bounded finite rank operator, $(\lambda-A)^{\nu} F_{2 i}$ is also a bounded finite rank operator even though $A$ is not bounded. Therefore, the sum in (1) is a compact operator. The lemma is now proven.

Lemma 1.3. Let $A$ bave a left quasi-inverse $A_{0}$, and a right quasi-inverse $A_{1}$; i.e., $A_{0} A=\left.\left(I+K_{1}\right)\right|_{D(A)}$ and $A A_{1}=I+K_{2}, K_{1}, K_{2} \in \mathcal{K}(X)$. Then $\left(A_{0}-A_{1}\right)$ $\epsilon \mathcal{K}(X)$.

Proof. $A_{0} A A_{1}=\left(I+K_{1}\right) A_{1}=A_{0}\left(I+K_{2}\right) \cdot A_{0}-A_{1}=\left(K_{1} A_{1}-A_{0} K_{2}\right) \in \mathcal{K}(X)$.

Lemma 1.4. Let $n$ be a given positive integer. Then for each $\lambda$ in $\left[\Phi_{A} \backslash \Phi^{0}(A)\right]$ except for at most an isolated set $\exists K_{\lambda} \in K(X)$, such that $\left[R_{\lambda}^{\prime}(A)\right]^{n}$ $+K_{\lambda}$ is a quasi-inverse of $(\lambda-A)^{n}$.

Proof. By Lemma 1.2, $R_{\lambda}^{\prime}(A)$ can be chosen so that $\left[R_{\lambda}^{\prime}(A)\right]^{n}$ is a right quasiinverse of $(\lambda-A)^{n}$. We shall refer to this particular choice of $R_{\lambda}^{\prime}(A)$ as $\left[R_{\lambda}^{\prime}(A)\right]_{n}$. By [3, Theorem 2.5], $(\lambda-A)^{n} \in \Phi(X)$. Therefore, it has a quasi-inverse, $S$. By Lemma 1.3, $S=\left[R_{\lambda}^{\prime}(A)\right]_{n}^{n}+K_{1}, K_{1} \in \mathcal{K}(X)$. By the same lemma, $R_{\lambda}^{\prime}(A)=\left[R_{\lambda}^{\prime}(A)\right]_{n^{+}}$ $K_{2}, K_{2} \in \mathcal{K}(X)$, when $\lambda$ is such that $R_{\lambda}^{\prime}(A)$ and $\left[R_{\lambda}^{\prime}(A)\right]_{n}$ are both defined. Note. $R_{\lambda}^{\prime}(A)$ and $\left[R_{\lambda}^{\prime}(A)\right]_{n}$ are both defined for all $\lambda \in \Phi_{A}$ except for at most an isolated set. Therefore,

$$
\left[R_{\lambda}^{\prime}(A)\right]^{n}=\left[R_{\lambda}^{\prime}(A)\right]_{n}^{n}+K_{3}, \quad K_{3} \in \mathcal{K}(X) .
$$


Therefore,

$$
\left[R_{\lambda}^{\prime}(A)\right]^{n}=S+K, \quad K \in K(X) \text { Q.E.D. }
$$

Lemma 1.5. Let $n$ be a positive integer and let a be such that $R_{\alpha}^{\prime}(A)$ and $\left[R_{\alpha}^{\prime}(A)\right]_{n}$ are both defined, where $\left[R_{\alpha}^{\prime}(A)\right]_{n}$ is as in the proof of Lemma 1.4. Let $f(\lambda)=(a-\lambda)^{-n}$. Then $\exists K \in \mathcal{K}(X)$ sucb that $f(A)+K$ is a quasi-inverse of $(\alpha-A)^{n}$.

Proof. By Theorem 9, $\S 2$, Lemma 1.1, and induction, we have $f(A)=\left[R_{\alpha}^{\prime}(A)\right]^{n}$ $+K_{1}, K_{1} \in \mathcal{K}(X)$. By Lemma 1.4 , there exists $K_{2} \in \mathcal{K}(X)$ such that $\left[R_{a}^{\prime}(A)\right]^{n}+K_{2}$ is a quasi-inverse of $(\alpha-A)^{n}$. Therefore, $f(A)+K$ is a quasi-inverse of $(\alpha-A)^{n}$, where $K=K_{2}-K_{1}$.

Theorem 1. Let $f(\lambda) \in \mathbb{Q}_{\infty}^{\prime}(A)$, and suppose either (a) that $f$ bas a zero of order $m$ at $\infty$, or (b) that $f$ vanishes identically in a neigbborbood of $\infty$. Let $P$ be a polynomial of degree $n$, where $0<n \leq m$ in case (a) and $0<n$ in case (b). Let $H(\lambda)=P(\lambda) f(\lambda)$. Then $H \in \mathbb{Q}_{\infty}^{\prime}(A), \exists K_{1}$ sucb that $R\left[f(A)+K_{1}\right] \subset D\left(A^{n}\right)$ and $\exists K_{2}$ such that $P(A)\left[f(A)+K_{1}\right]=\dot{H}(A)+K_{2} \cdot K_{1}, K_{2} \in \mathcal{K}(X)$.

Proof. Let $g(\lambda)=(\alpha-\lambda)^{n} f(\lambda)$, where $\alpha$ is as in Lemma 1.5.

$$
g(\lambda) \in \mathbb{Q}_{\infty}^{\prime}(A), \quad f(\lambda)=(\alpha-\lambda)^{-n} g(\lambda) .
$$

Let $b(\lambda)=(a-\lambda)^{-n}$. By Lemma 1.5, there exists a $K \in \mathcal{K}(X)$ such that $b(A)+K$ is a quasi-inverse of $(\alpha-A)^{n}$.

By Lemma 1.2, $R_{a}^{\prime}(A)$ can be chosen so that $(\alpha-A)^{i}\left[R_{a}^{\prime}(A)\right]^{i}=I+K_{i}^{\prime}$, $\forall i \leq n$. $K_{i}^{\prime} \in \mathcal{K}(X)$. We shall refer to this choice of $R_{\alpha}^{\prime}(A)$ as $\left[R_{\alpha}^{\prime}(A)\right]_{n}$. As shown in the proof of Lemma $1.5, \exists K \in \mathcal{K}(X)$ such that

$$
b(A)+K=\left[R_{\alpha}^{\prime}(A)\right]_{n}^{n} .
$$

By Theorem 9, $\$ 2 \exists K_{0} \in \mathcal{K}(X)$ such that

$$
\begin{aligned}
f(A)= & b(A) g(A)+K_{0} \\
= & (b(A)+K) g(A)-K g(A)+K_{0} \\
= & {\left[R_{\alpha}^{\prime}(A)\right]_{n}^{n} g(A)-K_{1}, \quad K_{1}=\left(K g(A)-K_{0}\right) \in K(X) . } \\
& \quad \therefore f(A)+K_{1}=\left[R_{a}^{\prime}(A)\right]_{n}^{n} g(A) .
\end{aligned}
$$

Let $G(\lambda)=(\alpha-\lambda)^{-n} P(\lambda) . G(\lambda) \in \mathbb{Q}_{\infty}^{\prime}(A)$ and $G(\lambda)=\sum_{i=0}^{n} a_{i}(\alpha-\lambda)^{i-n}$ where $P(\lambda)=\sum_{i=0}^{n} a_{i}(\alpha-\lambda)^{i}$. By Lemmas 1.5 and 1.3 , we have

$$
\begin{aligned}
G(A) & =\sum_{i=0}^{n} a_{i}\left\{\left[R_{\alpha}^{\prime}(A)\right]_{n}^{n-i}+K_{i 1}\right\} \\
& =\sum_{i=0}^{n} a_{i}\left[R_{\alpha}^{\prime}(A)\right]_{n}^{n-i}+K^{\prime} ; \quad K_{i 1}, K^{\prime} \in \mathcal{K}(X),
\end{aligned}
$$




$$
\begin{aligned}
P(A) & =\sum_{i=0}^{n} a_{i}(a-A)^{i}, \\
P(A)\left[R_{a}^{\prime}(A)\right]_{n}^{n} & =\sum_{i=0}^{n} a_{i}(a-A)^{i}\left[R_{a}^{\prime}(A)\right]_{n}^{n} \\
& =\sum_{i=0}^{n} a_{i}\left(I+K_{i}^{\prime}\right)\left[R_{\alpha}^{\prime}(A)\right]_{n}^{n-i}=\sum_{i=0}^{n} a_{i}\left[R_{\alpha}^{\prime}(A)\right]_{n}^{n-i}+K^{\prime \prime} \\
& =G(A)+K^{\prime \prime \prime}, \quad K_{i}^{\prime}, K^{\prime \prime}, K^{\prime \prime \prime} \in K(X), \\
\text {. } \quad P(A)\left(f(A)+K_{1}\right) & =P(A)\left[R_{\alpha}^{\prime}(A)\right]_{n}^{n} g(A) \\
=\left[G(A)+K^{\prime \prime}\right] g(A) & =G(A) g(A)+K_{0}^{\prime \prime}=(G \cdot g)(A)+K_{2}=H(A)+K_{2} .
\end{aligned}
$$

Definition 2. Let $X^{\prime}$ denote the set of all bounded linear functionals on $X$. A subset $S$ of $X^{\prime}$ is said to be total if the only element $x \in X$ which annihilates $S$ is the zero element of $X$.

At the beginning of $\$ 2$ it was explained that if $A \in \Phi(X)$, there exists an operator $T \in L(X)$ such that $T A=I-F_{1}$ and $A T=I-F_{2}$, where $F_{1}$ and $F_{2}$ are bounded finite rank operators.

Lemma 2.1. Let $n$ be any positive integer and let $A \in \Phi(X)$. Then $T$ can be chosen so that $F_{1} A^{j}$ is a bounded finite rank operator $\forall j \leq n$.

Proof. Since $A \in \Phi(X), A^{n} \in \Phi(X)$ with $D\left(A^{n}\right)$ dense in $X$. By [4, Chapter VII, Theorem 4.1], $D\left[\left(A^{n}\right)^{\prime}\right]$ is total in $X^{\prime}$. $\left(A^{n}\right)^{\prime}$ denotes the adjoint of $A^{n}$. Let $\left\{x_{i}\right\}_{i=1}^{k}$ be a basis for $N(A)$. Since $D\left[\left(A^{n}\right)^{\prime}\right]$ is total in $X^{\prime}, \exists x_{1}^{\prime} \in D\left[\left(A^{n}\right)^{\prime}\right] \ni x_{1}^{\prime}\left(x_{1}\right)$ $=1$. Let $x_{2} \in N(A) \cap N\left(x_{i}^{\prime}\right)$. $\exists \tilde{x}_{2}^{\prime} \in D\left[\left(A^{n}\right)^{\prime}\right] \ni \tilde{x}_{2}^{\prime}\left(x_{2}\right)=1$. Let $x_{2}^{\prime}=\tilde{x}_{2}^{\prime}-$ $\tilde{x}_{2}^{\prime}\left(x_{1}\right) x_{1}^{\prime}$. By induction there exist $k$ bounded linear functionals $\left\{x_{i}^{\prime}\right\}_{i=1}^{k}$ contained in $D\left[\left(A^{n}\right)^{\prime}\right] \ni x_{i}^{\prime}\left(x_{i}\right)=\delta_{i j}$. Letting $X_{1}=\bigcap_{i=1}^{k} N\left(x_{i}\right)$ we have that $X=N(A) \oplus X_{1}$. Letting $F_{1}$ be defined by $F_{1} x=\sum_{i=1}^{k} x_{i}^{\prime}(x) x_{i}$, we see that $T$ can be chosen so that $T A=\left.\left(I-F_{1}\right)\right|_{D(A)}$. Since $x_{i}^{\prime} \in D\left[\left(A^{n}\right)^{\prime}\right]$, and $D\left[\left(A^{n}\right)^{\prime}\right] \subset D\left[\left(A^{j}\right)^{\prime}\right]$ when $j \leq n$, we have $F_{1} A^{j} x=\Sigma_{i=1}^{k} x_{i}^{\prime}\left(A^{j} x\right) x_{i}=\sum_{i=1}^{k}\left[\left(A^{j}\right)^{\prime} x_{i}^{\prime}\right](x) x_{i}$ where $\left(A^{j}\right)^{\prime} x_{i}^{\prime}$ are bounded linear functionals. Therefore, $F_{1} A^{j}$ is a bounded finite rank operator.

Theorem 2. Let $n>0$ be given. There exists a bounded finite rank operator $F_{n}$ sucb that

$$
\left[R_{\lambda}^{\prime}(A)\right]^{n}(\lambda-A)^{n}=\left.\left(I-F_{n}\right)\right|_{D\left(A^{n}\right)}
$$

Proof. The following equation can be proven by induction in the same manner as the similar equation in the proof of Lemma 1.2.

$$
\left[R_{\lambda}^{\prime}(A)\right]^{n}(\lambda-A)^{n}=I-\sum_{j=0}^{n-1}\left[R_{\lambda}^{\prime}(A)\right]^{j}\left[\left(\lambda-\lambda_{i}\right) T_{i}+I\right]^{-1} F_{1 i}(\lambda-A)^{j}
$$


where $F_{1 i}$ is chosen as in Lemma 2.1. Since $F_{1 i} A^{j}$ is a bounded finite rank operator $\forall j \leq n$ we can take

$$
F_{n}=\sum_{j=0}^{n-1}\left[R_{\lambda}^{\prime}(A)\right]^{j}\left[\left(\lambda-\lambda_{i}\right) T_{i}+I\right]^{-1} F_{1 i}(\lambda-A)^{j} .
$$

Theorem 3. Let $D$ be an unbounded Caucby domain sucb that $\sigma_{\Phi}(A) \subset D$. Let $a \in \mathbf{C}$ be such that $\alpha \notin \bar{D}$. Then, if $P(\lambda)$ is a polynomial of degree $n$, there exists a compact operator, $K$, sucb that

$$
P(A) x=\frac{1}{2 \pi i} \int_{+B(D)} \frac{P(\lambda)}{(\lambda-\alpha)^{n+1}}(A-\alpha)^{n+1}\left[R_{\lambda}^{\prime}(A)\right]_{n+1} x d \lambda+K x
$$

for all $x \in D\left(A^{n}\right)$.

Proof. The following formula will be proven by induction.

(1) $(A-\alpha)^{n+1}\left[R_{\lambda}^{\prime}(A)\right]_{n+1} x=(\lambda-\alpha)^{n+1}\left[R_{\lambda}^{\prime}(A)\right]_{n+1} x-\sum_{k=0}^{n}(\lambda-\alpha)^{n-k}(A-\alpha)^{k} x+F x$ where $F$ is a bounded finite rank operator which depends on $n, \alpha, \lambda$, and $\left[R_{\lambda}^{\prime}(A)\right]_{n+1}$.

$$
(\lambda-A)\left[R_{\lambda}^{\prime}(A)\right]_{n+1} x=x-F_{0} x, \quad A\left[R_{\lambda}^{\prime}(A)\right]_{n+1} x=\lambda\left[R_{\lambda}^{\prime}(A)\right]_{n+1} x-x+F_{0} x .
$$

$F_{0}$ is a bounded finite rank operator.

$$
(A-\alpha)\left[R_{\lambda}^{\prime}(A)\right]_{n+1} x=(\lambda-\alpha)\left[R_{\lambda}^{\prime \prime}(A)\right]_{n+1} x-x+F_{0} x .
$$

Thus, formula ( 1 ) is true for $n=0$. Suppose (1) is true for $n-1$. Then

$$
(A-\alpha)^{n}\left[R_{\lambda}^{\prime}(A)\right]_{n+1} x=(\lambda-\alpha)^{n}\left[R_{\lambda}^{\prime}(A)\right]_{n+1} x-\sum_{k=0}^{n-1}(\lambda-\alpha)^{n-1-k}(A-\alpha)^{k} x+F_{1} x .
$$

Then

$$
\begin{aligned}
&(A-\alpha)^{n+1}\left[R_{\lambda}^{\prime}(A)\right]_{n+1} x \\
&=(\lambda-\alpha)^{n}(A-\alpha)\left[R_{\lambda}^{\prime}(A)\right]_{n+1}-\sum_{k=0}^{n-1}(\lambda-\alpha)^{n-1-\dot{k}}(A-\alpha)^{k+1} x+F_{2} x \\
&=(\lambda-\alpha)^{n}\left[(\lambda-\alpha)\left[R_{\lambda}^{\prime}(A)\right]_{n+1} x-x+F_{0} x\right] \\
&-\sum_{k=0}^{n-1}(\lambda-\alpha)^{n-1-k}(A-\alpha)^{k+1} x+F_{2} x \quad \text { by }(2) \\
&=(\lambda-\alpha)^{n+1}\left[R_{\lambda}^{\prime}(A)\right]_{n+1} x-(\lambda-\alpha)^{n} x+(\lambda-\alpha)^{n} F_{0} x \\
&-\sum_{k=0}^{n-1}(\lambda-\alpha)^{n-1-k}(A-\alpha)^{k+1} x+F_{2} x \\
&=(\lambda-\alpha)^{n+1}\left[R_{\lambda}^{\prime}(A)\right]_{n+1} x-\sum_{k=0}^{n}(\lambda-\alpha)^{n-k}(A-\alpha)^{k} x+F(x) .
\end{aligned}
$$

This completes the proof of (1) by induction. 


$$
\begin{aligned}
\text { - } \frac{1}{2 \pi i} & \int_{+B(D)} \frac{P(\lambda)}{(\lambda-\alpha)^{n+1}}(A-\alpha)^{n+1}\left[R_{\lambda}^{\prime}(A)\right]_{n+1} x d \lambda \\
= & \frac{1}{2 \pi i} \int_{+B(D)} \frac{P(\lambda)}{(\lambda-\alpha)^{n+1}}\left[(\lambda-\alpha)^{n+1}\left[R_{\lambda}^{\prime}(A)\right]_{n+1} x\right. \\
= & \left.\frac{1}{2 \pi i} \int_{+B(D)} P(\lambda)\left[R_{\lambda}^{\prime}(A)\right]_{n+1} x d \lambda \quad(\lambda-\alpha)^{n-k}(A-\alpha)^{k} x+F(\lambda) x\right] d \lambda \\
& -\frac{1}{2 \pi i} \int_{+B(D)} P(\lambda) \sum_{k=0}^{n}(\lambda-\alpha)^{-k-1}(A-\alpha)^{k} x d \lambda \\
& \quad-\frac{1}{2 \pi i} \int_{+B(D)} \frac{P(\lambda)}{(\lambda-\alpha)^{n+1}} F(\lambda) x d \lambda .
\end{aligned}
$$

Since $C(D)$ contains at most a finite number of points of $\Phi^{0}(A)$, the proof that the first of these integrals is compact is similar to the proof of Lemma $7.8, \S 2$. The last integral is compact because $F(\lambda)$ is compact for all $\lambda \in B(D)$. It remains only to consider the middle integral:

$$
B=-\frac{1}{2 \pi i} \int_{+B(D)} P(\lambda) \sum_{k=0}^{n}(\lambda-\alpha)^{-k-1}(A-\alpha)^{k} x d \lambda .
$$

Let $P(\lambda)=\sum_{i=0}^{n} a_{i}(\lambda-\alpha)^{2}$. $(1 / 2 \pi i) \int_{+B(D)} P(\lambda) /(\lambda-\alpha)^{k+1} d \lambda=-a_{k}$.

$$
\begin{aligned}
\text { - } B & =-\sum_{k=0}^{n} \frac{1}{2 \pi i} \int_{+B(D)} \frac{P(\lambda)}{(\lambda-\alpha)^{k+1}}(A-\alpha)^{k} x d \lambda \\
& =-\sum_{k=0}^{n}-a_{k}(A-\alpha)^{k} x=P(A) x . \quad \text { Q.E.D. }
\end{aligned}
$$

Theorem 4. Let $f \in \overrightarrow{\mathbb{U}}_{\infty}^{*}(A)$. Let $D$ be an unbounded Caucby domain such that $\sigma_{\Phi}(A) \subset D$ and $\bar{D} \subset \Delta(f)$. Let $\alpha \in \mathbf{C}$ be sucb that $\alpha \notin \bar{D}$. Then given any positive integer $n$, there exists a compact operator $K_{n}$ such that

$$
f(A) x=\frac{1}{2 \pi i} \int_{+B(D)} \frac{f(\lambda)}{(\lambda-\alpha)^{n+1}}(A-\alpha)^{n+1}\left[R_{\lambda}^{\prime}(A)\right]_{n+1} x d \lambda-K_{n} x \quad \forall x \in X .
$$

Proof.

$$
\begin{aligned}
& \frac{1}{2 \pi i} \int_{+B(D)} \frac{f(\bar{\lambda})}{(\lambda-\alpha)^{n+1}}(A-\alpha)^{n+1}\left[R_{\lambda}^{\prime}(A)\right]_{n+1} x d \lambda \\
&=\frac{1}{2 \pi i} \int_{+B(D)} f(\lambda)\left[R_{\lambda}^{\prime}(A)\right]_{n+1} x d \lambda \\
&-\sum_{k=0}^{n} \frac{1}{2 \pi i} \int_{+B(D)} f(\lambda)(\lambda-\alpha)^{-k-1}(A-\alpha)^{k} x d \lambda \\
&-\frac{1}{2 \pi i} \int_{+B(D)} \frac{f(\lambda)}{(\lambda-\alpha)^{n+1}} F(\lambda) x d \lambda, \quad F(\lambda) \in \mathcal{K}(X) .
\end{aligned}
$$


The proof of this statement is similar to the proof of the corresponding statement in the proof of Theorem 3.

$(1 / 2 \pi i) \int_{+B(D)}\left(f(\lambda) /(\lambda-\alpha)^{n+1}\right) F(\lambda) x d \lambda$ is compact because $F(\lambda)$ is compact for all $\lambda \in B(D)$. Since $a \notin \bar{D}$,

$$
\frac{1}{2 \pi i} \int_{+B(D)} f(\lambda)(\lambda-\alpha)^{-k-1}(A-\alpha)^{k} x d \lambda=\frac{1}{2 \pi i} \int_{+B\left(D_{1}\right)} \frac{f(\lambda)}{(\lambda-\alpha)^{k+1}}(A-\alpha)^{k} x d \lambda
$$

where $D_{1}$ is the infinite region of $D$.

$\therefore \frac{1}{2 \pi i} \int_{+B(D)} f(\lambda)(\lambda-\alpha)^{-k-1}(A-\alpha)^{k} x d \lambda=\frac{1}{2 \pi i} \int_{\Gamma} f(\lambda)(\lambda-\alpha)^{-k-1}(A-\alpha)^{k} x d \lambda$ where $\Gamma$ is a circle of radius $R>2|\alpha|$ lying in the outermost region of $D$.

$$
\left\|\frac{1}{2 \pi i} \int_{+B(D)} \frac{f(\lambda)}{(\lambda-\alpha)^{k+1}}(A-\alpha)^{k} x d \lambda\right\| \leq \frac{2^{k+1} \mu}{R^{k}}\left\|(A-\alpha)^{k} x\right\|
$$

where $|f(\lambda)| \leq \mu$ when $|\lambda| \geq R$. Such a $\mu$ exists because $f(\lambda) \in \mathbb{C}_{\infty}^{\prime}(A)$. Letting $R \rightarrow \infty$, we see that

$$
\frac{1}{2 \pi i} \int_{+B\left(D_{1}\right)} f(\lambda)(\lambda-\alpha)^{-k-1}(A-\alpha)^{k} x d \lambda=0, \quad \text { if } k>0 .
$$

If $k=0$, let $\mu=1 /(\lambda-\alpha)$ and let $g(\mu)=f(\lambda)$.

$$
\frac{1}{2 \pi i} \int_{+B\left(D_{1}\right)} \frac{f(\lambda)}{\lambda-\alpha} x d \lambda=\frac{1}{2 \pi i} \int_{\Omega} \frac{-g(\mu)}{\mu} x d \mu
$$

where $\Omega$ is the oriented image of $+B\left(D_{1}\right)$ under the mapping $\mu=1 /(\lambda-\alpha)$. Since $f(\lambda)$ is analytic on $D_{1}$ and at infinity, $g(\mu)$ is analytic in the interior of $\Omega$.

$$
\begin{aligned}
& \therefore \frac{1}{2 \pi i} \int_{+B\left(D_{1}\right)} \\
& \therefore \quad \frac{1}{(\lambda-\alpha)} x d \lambda=\frac{1}{2 \pi i} \int_{\Omega} \frac{-g(\mu)}{\mu} x d \mu=-g(0) x=-f(\infty) x \\
& \therefore+B(D) \frac{f(\lambda)}{(\lambda-\alpha)^{n+1}}(A-\alpha)^{n+1}\left[R_{\lambda}^{\prime}(A)\right]_{n+1} x d \lambda \\
&=\frac{1}{2 \pi i} \int_{+B(D)} f(\lambda)\left[R_{\lambda}^{\prime}(A)\right]_{n+1} x d \lambda+f(\infty) x+K_{n} x=f(A) x+K_{n} x .
\end{aligned}
$$

Theorem 5. Suppose $f \in \mathbb{Q}_{\infty}^{\prime}(A)$ and $f(\lambda) \neq 0$ for $\lambda \in \sigma_{\Phi}(A)$, but that $f(\infty)=0$, the zero at infinity being of finite order $m$. Then $\exists K_{1}, K_{2}$, and $K_{3} \in \mathcal{K}(X)$, and a closed operator B such that

$$
R\left(f(A)+K_{1}\right) \subset D\left(A^{m}\right)=D(B) \text { and } B\left(f(A)+K_{1}\right)=I+K_{2} .
$$

Furtber, for all $x \in D\left(A^{m}\right)$,

$$
B(x)=\frac{1}{2 \pi i} \int_{+B(D)}\left[f(\lambda)(\lambda-\alpha)^{m+1}\right]^{-1}(A-\alpha) R_{\lambda}^{\prime}(A)(\alpha-A)^{m} x d \lambda+K_{3} x,
$$

where $D$ is an unbounded Cauchy domain such that $\sigma_{\Phi}(A) \subset D, \bar{D} \subset \Delta(f)$, and $f(\lambda) \neq 0$ for $\lambda \in \bar{D}$. a is chosen so that $\alpha \notin \bar{D}$ and $\cdot\left[R_{\alpha}^{\prime}(A)\right]_{m}$ is defined.

Proof. Let $g(\lambda)=(\alpha-\lambda)^{m} f(\lambda) . g(\lambda) \in \mathbb{C}_{\infty}^{\prime}(A)$ and $g(\lambda)$ does not vanish on $\sigma_{\Phi}(A)$ or at infinity. 
Let $b(\lambda)=1 / g(\lambda) . \quad b(\lambda) \in \mathbb{Q}_{\infty}^{\prime}(A)$.

$$
\begin{aligned}
b(A) g(A) & =I+K_{4}, \\
g(A) b(A) & =I+K_{5}, \quad K_{4}, K_{5} \in \mathcal{K}(X), \\
f(\lambda) & =(\alpha-\lambda)^{-m} g(\lambda) .
\end{aligned}
$$

By Lemma 1.1, and Theorem 9 of $\$ 2, f(A)=\left[R_{a}^{\prime}(A)\right]_{m}^{m} g(A)-K_{1}, K_{1} \in \mathcal{K}(X)$. Let $B=b(A)(\alpha-A)^{m}$.

$$
\begin{aligned}
B\left(f(A)+K_{1}\right) & =b(A)(\alpha-A)^{m}\left[R_{\alpha}^{\prime}(A)\right]_{m}^{m} g(A) \\
& =b(A)\left(I-F_{1}\right) g(A), \quad \text { by Lemma } 1.2, \\
& =b(A) g(A)-F_{2}=I+K_{2}, \quad F_{1}, F_{2}, K_{2} \in \mathcal{K}(X) .
\end{aligned}
$$

To prove the second part of the theorem, we use Theorem 4, letting $n=0$, replacing $f(\lambda)$ by $b(\lambda)$, and replacing $x$ by $(\alpha-A)^{m} x$.

\section{The $\Phi$-Spectrum Mapping Theorem.}

Definition 1. $\hat{\sigma}(A)$ is defined as follows:

$$
\hat{\sigma}(A)= \begin{cases}\sigma(A) & \text { if } A \text { is bounded, } \\ \sigma(A) \cup(\infty) & \text { if } A \text { is unbounded. }\end{cases}
$$

In the standard operational calculus the Spectral Mapping Theorem states that $\sigma[f(A)]=f[\hat{\sigma}(A)]$.

Definition 2. $\hat{\sigma}_{\phi}(A)$ is defined as follows:

$$
\hat{\sigma}_{\Phi}(A)= \begin{cases}\sigma_{\Phi}(A) & \text { if } A \text { is bounded, } \\ \sigma_{\Phi}(A) \cup(\infty) & \text { if } A \text { is unbounded }\end{cases}
$$

In this section we will show that for our operational calculus $\sigma_{\Phi}[f(A)]=$ $f\left[\hat{\sigma}_{\Phi}(A)\right]$.

Lemma 1.1. Let $f \in \mathbb{A}_{\infty}^{\prime}(A)$ be sucb that $F(\lambda)=f(\lambda)(\alpha-\lambda) \in \mathbb{P}_{\infty}^{\prime}(A)$; Then $\exists K_{1}, K_{2}, K_{3} \in \mathcal{K}(X)$ sucb that $\left.\left(F(A)+K_{1}\right)\right|_{D(A)}=\left(f(A)-K_{2}\right)\left(I+K_{3}\right)(\alpha-A)$.

Proof. Let $\beta \in\left\{\Phi_{A} \backslash \Phi^{0}(A)\right\}$, let $g(\lambda)=f(\lambda)(\beta-\lambda)$, and let $G(\lambda)=$ $(\alpha-\lambda)(\beta-\lambda)^{-1} \cdot g(\lambda), G(\lambda) \in \mathbb{Q}_{\infty}^{\prime}(A)$.

$$
g(\lambda) G(\lambda)=F(\lambda)
$$

$$
\begin{aligned}
& \text { - } g(A) G(A)=F(A)+K_{4} \text { by Theorem 9, } 32, K_{4} \in K(X) \text {. } \\
& f(\lambda)=g(\lambda)(\beta-\lambda)^{-1} \text {. }
\end{aligned}
$$

By Lemma $1.1, \S 3$, and Theorem $9, \S 2$, 


$$
\begin{aligned}
f(A) & =g(A) R_{\beta}^{\prime}(A)+K_{2}, \quad K_{2} \in \mathcal{K}(X), \\
f(A)-K_{2} & =g(A) R_{\beta}^{\prime}(A), \\
\left(f(A)-K_{2}\right)(\beta-A) & =g(A) R_{\beta}^{\prime}(A)(\beta-A)=\left.g(A)\left(I+K_{5}\right)\right|_{D(A)}=\left.\left(g(A)+K_{6}\right)\right|_{D(A)} ; \\
\text { (2) }\left.\quad g(A)\right|_{D(A)} & =\left(f(A)-K_{2}\right)(\beta-A)-\left.K_{6}\right|_{D(A)} . \\
G(\lambda) & =(\alpha-\lambda)(\beta-\lambda)^{-1}=(\alpha-\beta)(\beta-\lambda)^{-1}+1 .
\end{aligned}
$$

$\therefore$ by Lemma 1.1 of $\$ 3$,

$$
G(A)=(\alpha-\beta) R_{\beta}^{\prime}(A)+I+K_{7}, \quad K_{7} \in \mathcal{K}(X) .
$$

$R_{\beta}^{\prime}(A)(\alpha-A)=R_{\beta}^{\prime}(A)(\alpha-\beta+\beta-A)=(\alpha-\beta) R_{\beta}^{\prime}(A)+\left.\left[I+K_{8}\right]\right|_{D(A)}, \quad K_{8} \in \mathcal{K}(X)$.

Therefore, by (3) we get

$$
\begin{gathered}
\left.G(A)\right|_{D(A)}=R_{\beta}^{\prime}(A)(\alpha-A)+\left.K_{9}\right|_{D(A)}, \quad K_{9} \in K(X), \\
\left.\left(G(A)-K_{9}\right)\right|_{D(A)}=R_{\beta}^{\prime}(A)(\alpha-A) .
\end{gathered}
$$

Note. $R\left\{\left.\left[G(A)-K_{9}\right]\right|_{D(A)}\right\} \subset D(A)$.

$$
\begin{aligned}
\left.g(A) G(A)\right|_{D(A)} & =g(A)\left[\left.\left(G(A)-K_{9}\right)\right|_{D(A)}+\left.K_{9}\right|_{D(A)}\right] \\
& =\left[\left(f(A)-K_{2}\right)(\beta-A)-K_{6}\right] R_{\beta}^{\prime}(A)(\alpha-A)+\left.g(A) K_{9}\right|_{D(A)}
\end{aligned}
$$

by (2) and (5)

$$
=\left(f(A)-K_{2}\right)(\beta-A) R_{\beta}^{\prime}(A)(\alpha-A)+\left.K_{10}\right|_{D(A)}, K_{10} \in K(X),
$$

because $R_{\beta}^{\prime}(A)(\alpha-A)$ is bounded.

$$
\begin{aligned}
\text { • }\left.g(A) G(A)\right|_{D(A)} & =\left(f(A)-K_{2}\right)\left(I+K_{3}\right)(a-A)+\left.K_{10}\right|_{D(A)} \\
& =\left.\left(F(A)+K_{4}\right)\right|_{D(A)} \text { by }(1) . \\
\text {. }\left.\quad\left(F(A)+K_{1}\right)\right|_{D(A)} & =\left(f(A)-K_{2}\right)\left(I+K_{3}\right)(a-A) \text { Q.E.D. }
\end{aligned}
$$

Theorem 1. If $f \in \mathbb{Q}_{\infty}^{\prime}(A)$, then $\sigma_{\Phi}[f(A)]=f\left[\hat{\sigma}_{\Phi}(A)\right]$.

Proof. First we show that $f\left[\hat{\sigma}_{\Phi}(A)\right] \subset \sigma_{\Phi}[f(A)]$. Suppose $\mu \in \sigma_{\Phi}(A)$. Let $g(\lambda)$ $=(f(\mu)-f(\lambda))(\mu-\lambda)^{-1}$ when $\lambda \neq \mu$, and let $g(\mu)=f^{\prime}(\mu)$. $g(\lambda) \in \mathbb{G}_{\infty}^{\prime}(A)$.

By Theorem 1, $\S_{3}, \exists K_{1}, K_{2} \in \mathcal{K}(X)$ such that $R\left(g(A)+K_{1}\right) \subset D(A)$ and

$$
(\mu-A)\left(g(A)+K_{1}\right)=f(\mu)-f(A)+K_{2} .
$$

By Lemma 1.1, $\exists K_{3}, K_{4}, K_{5} \in \mathcal{K}(X)$ such that

$$
\left.\left(f(\mu)-f(A)+K_{3}\right)\right|_{D(A)}=\left(g(A)-K_{4}\right)\left(I+K_{5}\right)(\mu-A) .
$$

Since $\mu \in \sigma_{\Phi}(A)$, either $\alpha(\mu-A)=\infty$ or $\beta(\mu-A)=\infty$, or $R(\mu-A)$ is not closed. 
By [2, Lemma 332], if $R(\mu-A)$ were not closed, $\beta(\mu-A)$ would be infinite. Therefore, either $a(\mu-A)=\infty$ or $\beta(\mu-A)=\infty$.

If $\alpha(\mu-A)=\infty$, then $a\left(f(\mu)-f(A)+K_{3}\right)=\infty$, by (2). Therefore, $(f(\mu)-Y(A)$ $\left.+K_{3}\right) \notin \Phi(X)$. Therefore, $(f(\mu)-f(A)) \notin \Phi(X)$ by [3, Theorem 2.8].

If $\beta(\mu-A)=\infty$, then $\beta\left(f(\mu)-f(A)+K_{2}\right)=\infty$. Therefore, $\left(f(\mu)-f(A)+K_{2}\right) \notin$ $\Phi(X)$ and $(f(\mu)-f(A)) \notin \Phi(X)$ by [3, Theorem 2.8].

$$
\text { . . } f(\mu) \in \sigma_{\Phi}[f(A)] \text {. }
$$

We will now show that $f(\infty) \in \sigma_{\Phi}\lfloor f(A)\rfloor$ if $A$ is unbounded. If $f(\infty)=f(\lambda)$ for some $\lambda \in \sigma_{\Phi}(A)$, then $f(\infty) \in \sigma_{\Phi}[f(A)]$ by what has already been proven. Therefore, we shall assume that $f(\infty)-f(\lambda) \neq 0$ for any $\lambda \in \sigma_{\Phi}(A)$. Let $g(\lambda)=f(\infty)-f(\lambda)$. Suppose $g(\lambda)$ has a zero of finite order $m$ at $\infty$. Then by Theorem $5, \S 3, \exists K \epsilon$ $\mathcal{K}(X)$ such that $R(g(A)+K) \subset D\left(A^{m}\right) \subset D(A)$.

Claim. $D(A)$ has infinite codimension.

Proof of Claim. As in the beginning of $\S 2, X=N\left(\lambda_{i}-A\right) \oplus X_{i}$, where $\operatorname{dim}\left[N\left(\lambda_{i}-A\right)\right]<\infty$ and $X_{i}$ is closed. We defined an operator $T_{i}$ such that $T_{i}$ is bounded and $R\left(T_{i}\right)=D(A) \cap X_{i}$. If $D(A) \cap X_{i}$ were closed, we would have $D(A)$ closed, because $D(A)=\left[D(A) \cap X_{i}\right] \oplus N(A)$. But $D(A)$ is not closed, because $A$ is unbounded. Therefore, $D(A) \cap X_{i}$ is not closed. Therefore, by [2, Lemma 332], $D(A) \cap X_{i}$ has infinite codimension. Since $D(A)=\left[D(A) \cap X_{i}\right] \oplus N\left(\lambda_{i}-A\right)$, and $\operatorname{dim} N\left(\lambda_{i}-A\right)<\infty$, we see that $D(A)$ has infinite codimension. This completes the proof of the claim.

Since $R(g(A)+K) \subset D(A)$, we have that $\beta(g(A)+K)=\infty$. Therefore, $(g(A)+$ $K) \notin \Phi(X)$. $\quad \therefore g(A) \notin \Phi(X)$ by [3, Theorem 2.8].

We shall now suppose that $g(\lambda)=f(\lambda)-f(\infty)$ has a zero of infinite order at $\infty$. This means that $g(\lambda)$ is zero on a neighborhood of $\propto$. Since $g(\lambda) \neq 0$ for any $\lambda \epsilon$ $\sigma_{\Phi}(A), \sigma_{\Phi}(A)$ must be bounded.

Let $b(\lambda)$ be defined in the following way. Let $C$ be a circle that contains $\sigma_{\Phi}(A)$ and the point $\lambda_{i}$ in its interior. Then

$$
b(\lambda)=\left\{\begin{array}{l}
1 \text { if } \lambda \text { is in the interior of } C, \\
0 \text { if } \lambda \text { is in the exterior of } C,
\end{array}\right.
$$

3) $\quad b(A) g(A)=g(A)+K_{1}$,

$g(A) b(A)=g(A)+K_{2}, \quad K_{1}, K_{2} \in \mathcal{K}(X)$,

$$
b(A)=\frac{1}{2 \pi i} \int_{C} R_{\lambda}^{\prime}(A) d \lambda=\frac{1}{2 \pi i} \int_{C} T_{i}\left[\left(\lambda-\lambda_{i}\right) T_{i}+I\right]^{-1} d \lambda .
$$

By the same argument used in the proof of Lemma 7.4, $\$ 2$, we have

$$
b(A)=\frac{1}{2 \pi i} \int_{\Gamma} \frac{-1}{\mu} I d \mu+\frac{1}{2 \pi i} \int_{\Gamma}\left(\mu+T_{i}\right)^{-1} d \mu
$$

where $\Gamma$ is the image of $C$ under the mapping $\mu=1 /\left(\lambda-\lambda_{i}\right)$. 
$R\left(T_{i}\right) \subset D(A) \neq X$ because $A$ is unbounded. Therefore,

$$
0 \in \sigma_{\Phi}\left(T_{i}\right) \subset \sigma\left(T_{i}\right) \text { because } \beta\left(T_{i}\right)=\infty .
$$

Since $\lambda_{i}$ is in the interior of $C$, zero will be in the interior of $\Gamma$, and $\Gamma$ will have a negative orientation. Then $b(A)=I-(1 / 2 \pi i) \int_{-\Gamma}\left[\mu-\left(-T_{i}\right)\right]^{-1} d \mu$.

By (5), $0 \in \sigma_{\Phi}\left(-T_{i}\right) \subset \sigma\left(-T_{i}\right) . b(A)$ is thus seen to be the projection associated with a spectral set of $-T_{i}, \sigma_{1}\left(-T_{i}\right)$, which does not contain zero. For a discussion of spectral sets and projections, see [5, p. 298]. Since $0 \in \sigma_{\Phi}\left(-T_{i}\right)$, we see by what has already been proven that $b(A) \notin \Phi(X)$. Therefore, $\alpha(b(A))=\infty$ or $\beta(b(A))=\infty$. Using equalities (3) and (4), we see that $g(A) \notin \Phi(X)$, or $f(\infty) \epsilon$ $\sigma_{\Phi}[f(A)]$. The proof that $f\left[\hat{\sigma}_{\phi}(A)\right] \subset \sigma_{\Phi}[f(A)]$ is now complete.

We proceed to prove that $\sigma_{\Phi}[f(A)] \subset f\left[\hat{\sigma}_{\Phi}(A)\right]$.

Let $\beta \in \sigma_{\Phi}[f(A)]$. Suppose $A$ is bounded and $\beta \notin f\left[\sigma_{\Phi}(A)\right]$.

Let $b(\lambda)=1 /(\beta-f(\lambda)), b(\lambda) \in \mathbb{Q}^{\prime}(A)$.

By Theorem 11, $\left\{2, f(A)=f^{*}(A)\right.$. Therefore, $b^{*}(A)[\beta-f(A)]=I+K_{1}$, $[\beta-f(A)] b^{*}(A)=I+K_{2}, K_{1}, K_{2}, \in \mathcal{K}(X)$.

Therefore, by [3, Lemma 2.4], $\beta \in \Phi[f(A)]$. Since this contradicts the assumption that $\beta \in \sigma_{\Phi}[f(A)]$, we must conclude that $\beta \in f\left[\sigma_{\Phi}(A)\right]$.

If $A$ is unbounded and $\beta \notin f\left[\hat{\sigma}_{\Phi}(A)\right]$, then $b(\lambda)=1 /(\beta-f(\lambda)) \in \mathbb{G}_{\infty}^{\prime}(A)$.

$$
b(A)[\beta-f(A)]=I+K_{1}, \quad[\beta-f(A)] b(A)=I+K_{2}, \quad K_{1}, K_{2} \in \mathcal{K}(X) .
$$

We conclude that $\beta \in f\left[\hat{\sigma}_{\Phi}(A)\right]$ just as we did when $A$ was bounded. Therefore, $\sigma_{\Phi}[f(A)] こ f\left[\hat{\sigma}_{\Phi}(A)\right]$. Q.E.D.

Theorem 2. Suppose $f \in \mathbb{Q}_{\infty}^{\prime}(A)$ and $g \in \mathbb{Q}^{\prime}[f(A)]$. Define $F$ by $F(\lambda)=g[f(\lambda)]$ for $f(\lambda) \in \Delta(g)$. Then $F \in \mathbb{G}_{\infty}^{\prime}(A)$ and $F(A)=g[f(A)]+K, K \in \mathcal{K}(X)$.

Proof. By Theorem 1, $\hat{\sigma}_{\Phi}(A) \subset \Delta(F)$. Therefore, $F \in \mathbb{P}_{\infty}^{\prime}(A)$.

Let $D$ be a bounded Cauchy domain such that $\sigma_{\Phi}[f(A)] \subset D$ and $\bar{D} \subset \Delta(g)$. Let $D_{1}$ be an unbounded Cauchy domain such that $\sigma_{\Phi}(A) \subset D_{1}, \bar{D}_{1} \subset \Delta(f)$, and $f\left(\bar{D}_{1}\right) \subset D$. Then, if $A$ is unbounded, $F(A)=F(\infty) I+(1 / 2 \pi i) \int_{+B\left(D_{1}\right)}[f(\xi)] R_{\xi}^{\prime}(A) d \xi$.

$$
g[f(\xi)]=\frac{1}{2 \pi i} \int_{+B(D)} \frac{g(\lambda)}{\lambda-f(\xi)} d \lambda
$$

Let $h_{\lambda}(\xi)=[\lambda-f(\xi)]^{-1}$, where $\lambda$ is fixed on $B(D)$.

$b_{\lambda}(A)[\lambda-f(A)]=I+K_{1}(\lambda), \quad[\lambda-f(A)] b_{\lambda}(A)=I+K_{2}(\lambda), \quad K_{1}(\lambda), K_{2}(\lambda) \in \mathcal{K}(X)$.

By Lemma 1.3, \$3, $b_{\lambda}(A)=R_{\lambda}^{\prime}[f(A)]+K_{3}(\lambda), K_{3}(\lambda) \in \mathcal{K}(X) . K_{3}(\lambda)$ is continuous in $\lambda$ on $B(D)$, because $b_{\lambda}(A)$ and $R_{\lambda}^{\prime}[f(A)]$ are continuous in $\lambda$ on $B(D)$. 


$$
\begin{aligned}
F(A) & =\frac{1}{2 \pi i} \int_{+B(D)} \frac{g(\lambda)}{\lambda-f(\infty)} d \lambda I+\frac{1}{2 \pi i} \int_{+B\left(D_{1}\right)} \frac{1}{2 \pi i} \int_{+B(D)} \frac{g(\lambda)}{\lambda-f(\xi)} d \lambda R_{\xi}^{\prime}(A) d \xi \\
& =\frac{1}{2 \pi i} \int_{+B(D)} g(\lambda) b_{\lambda}(\infty) d \lambda I+\frac{1}{2 \pi i} \int_{+B(D)} g(\lambda) \frac{1}{2 \pi i} \int_{+B\left(D_{1}\right)} b_{\lambda}(\xi) R_{\xi}^{\prime}(A) d \xi d \lambda \\
& =\frac{1}{2 \pi i} \int_{+B(D)} g(\lambda) b_{\lambda}(\infty) d \lambda I+\frac{1}{2 \pi i} \int_{+B(D)} g(\lambda)\left[b_{\lambda}(A)-b_{\lambda}(\infty) I\right] d \lambda \\
& =\frac{1}{2 \pi i} \int_{+B(D)} g(\lambda) b_{\lambda}(A) d \lambda=\frac{1}{2 \pi i} \int_{+B(D)} g(\lambda)\left[R_{\lambda}^{\prime}[f(A)]+K(\lambda)\right] d \lambda \\
& =g[f(A)]+K, \quad K \in \mathcal{K}(X) .
\end{aligned}
$$

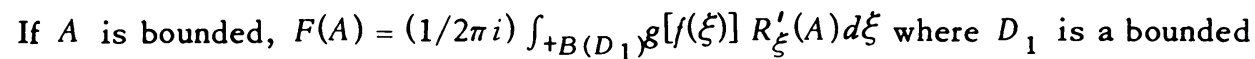
Cauchy domain. The rest of the proof proceeds as above. Q.E.D.

5. An analytic basis. In [2, Theorem 3], it is shown that if $\lambda_{i} \epsilon$ $\left\{\Phi_{i}(A) \backslash S_{i}(A)\right\}$, there exist $n$ vector valued functions, $\left\{x_{i}(\lambda)\right\}_{i=1}^{n}$, analytic in some neighborhood of $\lambda_{i}$, which form a basis for $N(A-\lambda)$. We proceed to show that the functions, $\left\{x_{i}(\lambda)\right\}_{i=1}^{n}$, can be chosen so that they are analytic in all of $\left\{\Phi_{i}(A) \backslash S_{i}(A)\right\}$ except for at most a countable set of points having no accumulation point in $\Phi_{i}(A)$.

Theorem 1. Let $\Phi_{i}(A)$ be a component of $\Phi_{A}$, and let $\lambda_{i}$ be any point of $\left\{\Phi_{i}(A) \backslash S_{i}(A)\right\}$, where $S_{i}(A)$ is as in Theorem $1, \S 2$. Let $x_{1}, \cdots, x_{n}$ be a bas is for $N\left(A-\lambda_{i}\right)$. Then there exist vector valued functions, $x_{1}(\lambda), \ldots, x_{n}(\lambda)$, analytic for all $\lambda \in \Phi_{i}(A)$ except for at most a countable set of points baving no accumulation point in $\Phi_{i}(A)$, such that $x_{j}\left(\lambda_{i}\right)=x_{j}, 1 \leq j \leq n$, and $\left\{x_{j}(\lambda)\right\}_{j=1}^{n}$ form a basis for $N(A-\lambda)$.

Proof. Define the operator $R_{\lambda}^{\prime}(A)$ on $\Phi_{i}(A)$ as in Definition $8, \S 2$, and let $\lambda_{i}$ be the point used in the definition. $R_{\lambda}^{\prime}(A)=T_{i}\left[\left(\lambda-\lambda_{i}\right) T_{i}+i\right]^{-1}$. Let $\lambda \epsilon$ $\left\{\Phi_{i}(A) \backslash \Phi_{i}^{0}(A)\right\}$ and let $x \in N(A-\lambda)$.

$$
\begin{aligned}
R_{\lambda}^{\prime}(A)(\lambda-A) x & =x-\left[\left(\lambda-\lambda_{i}\right) T_{i}+I\right]^{-1} F_{1 i} x=0, \\
x & =\left[\left(\lambda-\lambda_{i}\right) T_{i}+I\right]^{-1} F_{1 i} x .
\end{aligned}
$$

Let $F_{1 i} x=\sum_{j=1}^{n} f_{j}(x) x_{j}$, where $\left\{f_{j}\right\}_{j=1}^{n}$ are bounded linear functionals on $X$. Now,

$$
x=\sum_{j=1}^{n} f_{j}(x)\left\{\left[\left(\lambda-\lambda_{i}\right) T_{i}+I\right]^{-1} x_{j}\right\} .
$$

Therefore, the $n$ vectors, $\left[\left(\lambda-\lambda_{i}\right) T_{i}+I\right]^{-1} x_{j}, 1 \leq j \leq n$, span $N(A-\lambda)$ and since $\alpha(A-\lambda)=n$, they must be linearly independent. Therefore, the vectors, $x_{j}(\lambda)=$ $\left[\left(\lambda-\lambda_{i}\right) T_{i}+I\right]^{-1} x_{j}, 1 \leq j \leq n$, form a basis for $N(A-\lambda)$. We also have that $x_{j}\left(\lambda_{i}\right)=\left[\left(\lambda_{i}-\lambda_{i}\right) T_{i}+I\right]^{-1} x_{j}=x_{j}$. Q.E.D. 


\section{BIBLIOGRAPHY}

1. I. C. Gohberg and M. G. Kreìn, The basic propositions on defect numbers, root numbers and indices of linear operators, Uspehi Mat. Nauk 12 (1957), no. 2 (74), 43-118; English transl., Amer. Math. Soc. Transl. (2) 13 (1960), 185-264. MR 20 \#3459; MR 22 \#3984.

2. Tosio Kato, Perturbation theory for nullity, deficiency and other quantities of linear operators, J. Analyse Math. 6 (1958), 261-322. MR 21 \#6541.

3. Martin Schechter, Basic theory of Fredholm operators, Ann. Scuola Norm. Sup. Pisa (3) 21 (1967), 261-280. MR 36 \#6977.

4. - - Principles of functional analysis, Academic Press, New York, 1971.

5. A. E. Taylor, Introduction to functional analysis, Wiley, New York; Chapman \& Hall, London, 1958. MR 20 \#5411.

6. - Spectral theory of closed distributive operators, Acta Math. 84 (1951), 189224. MR 12, 717.

DEPARTMENT OF MATHEMATICS, BERNARD M. BARUCH COLLEGE (CUNY), NEW YORK, NEW YORK 10010

BELFER GRADUATE SCHOOL OF SCIENCE, YESHIVA UNIVERSITY, NEW YORK, NEW YORK 10033 